Draft version August 14, 2018

Preprint typeset using IATEX style emulateapj v. 05/12/14

\title{
THE ROLE OF FAST MAGNETIC RECONNECTION ON THE RADIO AND GAMMA-RAY EMISSION FROM THE NUCLEAR REGIONS OF MICROQUASARS AND LOW LUMINOSITY AGNS
}

\author{
L.H.S. Kadowaki ${ }^{1}$, E.M. De Gouveia Dal Pino ${ }^{1}$, And Chandra B. Singh ${ }^{1}$ \\ Draft version August 14, 2018
}

\begin{abstract}
Fast magnetic reconnection events can be a very powerful mechanism operating in the core region of microquasars and AGNs. In earlier work, it has been suggested that the power released by fast reconnection events between the magnetic field lines lifting from the inner accretion disk region and the lines anchored into the central black hole could accelerate relativistic particles and produce the observed radio emission from microquasars and low luminosity AGNs (LLAGNs). Moreover, it has been proposed that the observed correlation between the radio emission and the mass of these sources, spanning $10^{10}$ orders of magnitude in mass, might be related to this process. In the present work, we revisit this model comparing two different fast magnetic reconnection mechanisms, namely, fast reconnection driven by anomalous resistivity (AR) and by turbulence (as described in Lazarian \& Vishiniac 1999). We apply the scenario above to a much larger sample of sources (including also blazars, and gamma-ray bursts - GRBs), and find that LLAGNs and microquasars do confirm the trend above. Furthermore, when driven by turbulence, not only their radio but also their gamma-ray emission can be due to magnetic power released by fast reconnection, which may accelerate particles to relativistic velocities in the core region of these sources. Thus the turbulent-driven fast reconnection model is able to reproduce better the observed emission than the AR model. On the other hand, the emission from blazars and GRBs does not follow the same trend as that of the LLAGNs and microquasars, suggesting that the radio and gamma-ray emission in these cases is produced further out along the jet, by another population of relativistic particles, as expected.

Keywords: accretion, accretion disks - galaxies: active - gamma rays: general - magnetic reconnection - radio continuum: general - X-rays: binaries
\end{abstract}

\section{INTRODUCTION}

Galactic black hole binary systems (also referred as microquasars) and active galactic nuclei (AGNs) often exhibit variability and quasi-periodic relativistic outflow ejections of matter that may offer important clues about the physical processes that occur in their inner regions, in the surroundings of the central black hole $(\mathrm{BH})$. Several authors have been exploring for decades these phenomena both observationally and also by means of theoretical and numerical modelling (see, e.g., de Gouveia Dal Pino \& Lazarian 2005; Remillard \& McClintock 2006; McKinnev \& Blandford 2009; Fender \& Belloni 2012; Zhang et al. 2014, for recent reviews).

A potential model to explain the origin of these ejections and the often associated radio flare emissions was proposed by de Gouveia Dal Pino \& Lazarian (2005, hereafter GL05) for microquasars and extended to AGNs and young stellar objects by de Gouveia Dal Pino et al. (2010a, herafter GPK10) (see also de Gouveia Dal Pino et al. 2010b). Their model invokes the interactions between the magnetosphere anchored into the central BH horizon (Blandford \& Znajek 1977) and the magnetic field lines arising from the accretion disk.

In accretion episodes where the accretion rate is increased (and may even approach the critical Eddington rate), both magnetic fluxes are pushed to-

\footnotetext{
${ }^{1}$ Department of Astronomy (IAG-USP), University of Sao Paulo, Brazil; luis.kadowaki@iag.usp.br; dalpino@iag.usp.br
}

gether in the inner disk region and reconnect under finite magnetic resistivity (see Figure 11). In the presence of kinetic plasma instabilities (Shav et al. 2004; Yamada et al. 2010), anomalous resistivity (e.g., Parker 1979; Biskamp et al. 1997; Shav et al. 1998), or turbulence (see Kowal et al. 2009, 2012; Lazarian \& Vishiniac 1999, hereafter LV99), reconnection becomes very efficient and fast (with reconnection velocities approaching the local Alfvén speed, which in these systems is near the light speed) and then may cause the release of large amounts of magnetic energy power. Part of this power will heat the coronal and the disk gas and part may accelerate particles to relativistic velocities.

A first-order Fermi process for particle acceleration at the magnetic discontinuity was first described analytically in GL05 and then successfully tested numerically in collisionless pair plasmas by means of particle in cell simulations which can probe only the kinetic scales of this process (see Drake et al. 2006, 2010; Zenitani \& Hoshino 2008; Zenitani et al. 2009)2, and more recently by means of 3D collisional MHD simulations with injected test particles which probed the efficiency of this process also at the macroscopic scales of the flow by Kowal et al. (2011, 2012) (see also de Gouveia Dal Pino \& Kowal 2015; de Gouveia Dal Pino et al. 2014, for reviews). It has been found that particles accelerated by this process are able to produce Synchrotron radio spectra with power-law indices that are comparable to the observa-

2 Where magnetic islands or Petcheck-like X-point configurations of fast reconnection are naturally driven and sustained by, e.g., the Hall effect or kinetic instabilities 


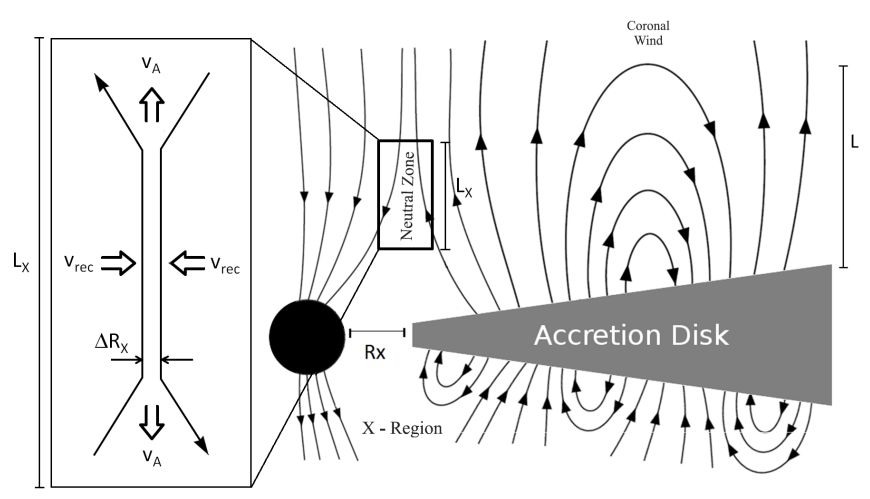

Figure 1. Schematic drawing of the magnetic field geometry in the region surrounding the $\mathrm{BH} . R_{X}$ characterizes the inner accretion disk radius where the disk ram pressure balances the $\mathrm{BH}$ magnetosphere pressure. The lines arising from the accretion disk into the corona are pushed towards those anchored into the $\mathrm{BH}$ when the accretion rate is large enough and reconnection is fast at the magnetic discontinuity region of width $\Delta R_{X}$ and extension $L_{X}$ in the Figure (adapted from GL05). In the detail on the left side we highlight the magnetic reconnection site properties. In particular, when turbulence is present, this is superposed to the large scale magnetic field lines.

tions (Kowal et al. 2012; de Gouveia Dal Pino \& Kowal 2015; de Gouveia Dal Pino et al. 2014; del Valle et al. $2014]^{3}$.

Employing the magnetic reconnection model above in the surrounds of BHs, GPK10 found some evidence that the observed correlation between the radio luminosities and the $\mathrm{BH}$ source masses, spanning $10^{10}$ orders of magnitude in mass and $10^{6}$ in luminosity, from microquasars to low-luminosity AGNs (LLAGNs) (see Merloni et al. 2003; Nagar et al. 2005; Fender et al. 2004) could be explained by the magnetic power released by fast reconnection. They also argued that this mechanism could be related to the transition between the observed "hard" and "soft" steep-power-law (SPL) states of microquasars (e.g., Remillard \& McClintock 2006).

Lately, similar mechanisms involving magnetic activity and reconnection in the core regions of compact sources to explain their emission spectra have been also invoked by other authors (see, e.g., Igumenshchev 2009; Soker 2010; Uzdensky \& Spitkovsky 2014; Dexter et al. 2014; Huang et al.|2014). In particular, magnetic reconnection between the magnetospheric lines of the central source and those anchored into the accretion disk resulting in the ejection of plasmons has been detected in numerical MHD studies (see, e.g., Romanova et al. 2002, 2011; Zanni \& Ferreira 2009, 2013; Cemeliić et al. 2013). The recent numerical relativistic MHD simulations of magnetically arrested accretion disks by Tchekhovskoy et al. (2011); McKinney et al. (2012) and Dexter et al. (2014) also evidence the development of magnetic reconnection in the magnetosphere of the $\mathrm{BH}$ and are consistent with the scenario above. Dexter et al. (2014) also suggest that

\footnotetext{
3 As argued in GL05, the particle acceleration mechanism above is not the only possibility. Relativistic particles may be also produced behind shocks in the surrounds of the reconnection region. As in the Sun, plasmoids formed by reconnection of the field lines may violently erupt and cause the formation of a shock front and accelerated particles behind the shock front can also lead to powerlaw Synchrotron radio emission.

4 By LLAGNs we mean sources with $L_{H \alpha} \leq 10^{40} \mathrm{erg} \mathrm{s}^{-1}$ (see Ho et al. 1997; Nagar et al. 2005).
}

this process could be related to the transition from the "hard" to the "soft" SPL states and the transient phenomena observed in $\mathrm{BH}$ binaries.

In the framework of very high energy (VHE) emission, for several years blazars (FSRQs and BL Lac objects), i.e., AGNs with highly beamed jets towards the line of sight, have been the largest sample detected by gammaray telescopes. This fact is consistent with the standard scenario for which the VHE of these sources is attributed to conventional relativistic particle acceleration along the jet, with the gamma-ray emission (due to either leptonic synchrotron self-Compton - SSC, or proton synchrotron, or Inverse Compton emission) being strongly Doppler boosted and producing the observed apparently high fluxes.

Recently, however, a few nearby radio galaxies, which are considered LLAGNs, have been also detected at TeV energies by HEGRA, HESS, MAGIC and VERITAS, namely M87, Cen A, and NGC1275 (see, e.g., Sol et al. 2013, and references therein) 5 . The viewing angle of the large scale jets of these sources is of several degrees (e.g., for M87 it is of the order of $30^{\circ}$; Reynoso et al. 2011), which allows only moderate Doppler boosting and the AGN source is highly underluminous (e.g., for M87, $\left.L_{b o l} \leq 10-5 L_{E d d}\right)$. Both characteristics make it very hard to explain the VHE of these sources within the same standard scenario of blazars. Besides, the $\mathrm{TeV}$ emission in these sources is highly variable with timescales of a few days ( $t_{v a r} \sim 1-2$ days for M87), pointing to extremely compact emission regions (corresponding to scales of only a few Schwarzschild radii; e.g., Abramowski et al. 2012). These findings have motivated several new studies on alternative particle acceleration mechanisms (see, e.g., de Gouveia Dal Pino et al. 2014, for reviews) and emission theories (e.g., Neronov \& Aharonian 2007; Rieger \& Aharonian 2008; Tavecchio \& Ghisellini 2008a, b; Abdo et al. 2009b), where gamma-ray GeV-TeV emission is considered to be produced in the vicinity of the $\mathrm{BH}$ (in a pulsar-like cascade mechanism) and/or at the jet launching basis.

As described above, magnetic activity and reconnection events occurring close to $\mathrm{BHs}$ could also offer appropriate conditions for producing particle acceleration and the associated VHE gamma-ray emission in these sources, via interactions of the accelerated particles with the photon, density and magnetic fields in the surrounds of the BH. Similarly, microquasars are also expected to emit high energy gamma-rays, particularly owing to their general similarities to LLAGNs (e.g., Romero et al. 2007, GPK10). Until now, only one source of this type, namely $\mathrm{Cyg}-\mathrm{X} 3$, has been unambiguously detected in the GeV gamma-rays, by the Agile and Fermi observatories (Tavani et al. 2009; Abdo et al. 2009a). At TeV energies, only upper limit fluxes are available, in spite of intensive monitoring (Aleksić et al. 2010b). There is also some evidence of sporadic GeV-TeV gamma-ray emission from Cyg X-1 (Albert et al. 2007; Malyshev et al. 2013) and upper limits in the $0.1-10 \mathrm{GeV}$ range for GRS $1915+105$ and GX 339 - 4 (see, e.g., Bodaghee et al. 2013).

With regard to blazars, a recent study has identified an

\footnotetext{
5 There is also the case of IC310, a radio galaxy which has been interpreted as belonging to the blazar family, and a few other cases of radio galaxies to be confirmed at $\mathrm{VHE}$
} 
important correlation between these sources and gammaray bursts (GRBs) (see Nemmen et al. 2012). These authors showed that the relativistic jets produced in both classes exhibit nearly the same correlation between the kinetic power carried out by accelerated particles and the gamma-ray luminosity. They concluded that this would be an indication that the bulk of the high energy emission comes from the jet region in these sources, therefore reinforcing the standard scenario for blazar emission. On the other hand, so far there has been no evidence of such a correlation for LLAGNs. This lack of correlation may be a further indication that the acceleration zones responsible for the observed high energy emission in the latter are not in the jet, but in the core, at the jet launching region, as suggested in the GL05 (and GPK10) model and by the observations described above.

Since the gamma-ray emission is correlated with the accelerated relativistic particles that produce the radio observed emission (via, e.g., Inverse Compton, Synchrotron self-Compton, proton-proton, or proton-photon up-scatterings), in the present work we apply the GL05 scenario of reconnection-driven acceleration in the magnetized corona around the accretion disk and the $\mathrm{BH}$ of the sources and investigate whether or not the gammaray emission of microquasars and LLAGNs can be also associated to this mechanism in the core region. Moreover, we explore two possible mechanisms for driving fast magnetic reconnection, namely, anomalous resistivity which was also employed in the previous GL05 and GPK10 studies, and turbulence based on LV99 model. We further extend the earlier study by GPK10, who found a correlation between the observed radio luminosity of the accelerated particles and the magnetic power released by reconnection in the core region for a few microquasars and LLAGNs, to a much larger sample of sources. We find that this trend is confirmed also in the much larger sample. We finally compare this with a sample of blazars and GRBs to examine the trend for higher luminous sources.

The sections of this work are divided as follows: in section 2 we briefly describe the model and summarize the main assumptions used to calculate the magnetic power released by fast magnetic reconnection in the core region of compact sources hosting BHs, comparing the results obtained for assuming either anomalous resistivity or turbulence to make reconnection fast. In section 3, we describe the sample of sources and their observed core radio and gamma-ray emission which are compared with the theoretical predictions of our model. Finally, in section 4, we discuss the implications of our results for all classes of sources and draw our general conclusions.

\section{MAGNETIC POWER PRODUCED BY FAST RECONNECTION IN THE SURROUNDS OF A BH}

\subsection{Possible scenario for the accretion disk and corona right before fast magnetic reconnection}

Our model is described in detail in earlier work (GL05 and GPK10) and we summarize here its main assumptions introducing important new upgrades as described below. As in GL05 and GPK10, we assume that the inner region of the accretion disk/corona system alternates between two states which are controlled by changes in the global magnetic field. As described in the previous section, we consider that right before a fast magnetic recon- nection event the system is in a state that possibly characterizes the transition from the hard to the soft state, and adopt a magnetized standard (geometrically thin and optically thick) accretion disk (Shakura \& Sunvaev 1973) with a corona around a BH, as shown in the cartoon of Figure 1. Nevertheless, we must stress that the real structure of the accretion disk is not a crucial point for the purposes of our study because our focus is the inner coronal disk region where the interaction of the magnetic field lines with the $\mathrm{BH}$ magnetosphere takes place (see more in the Discussion section).

A magnetosphere around the central BH may be built from the continuous drag of magnetic field lines by the accretion disk (e.g., Macdonald et al. 1986; Wang et al. 2002). The coronal disk large-scale poloidal magnetic field can be in turn established either by advection of lines carried from the outer regions of the disk or by the action of a dynamo inside the accretion disk (e.g., Livio et al. 2003; King et al. 2004; Uzdensky \& Goodman 2008; Krolik \& Piran 2011, 2012) possibly driven by the combination of magnetorotational instability (see Balbus \& Hawley 1998) and disk differential rotation.

According to mean field dynamo theory (see GL05 and references therein, an inversion of the polarization of the large scale magnetic lines is expected to occur every half of the dynamo cycle; when this happens a new flux of disk lines should reach the inner region with an inverted polarity with respect to the magnetic flux already anchored into the $\mathrm{BH}$, therefore, favouring magnetic reconnection between the two fluxes (GL05, GPK10). Whether the magnetic flux is produced by a dynamo in the disk itself or advected from the outer regions, or both, its sign is expected to change periodically, although the characteristic time scale of this variation is hard to compute in the absence of a detailed modelling of the dynamo in the disk or of the flux advected from the outer regions (Tagger et al. 2004; Tchekhovskov et al. 2014, GL05). In the case of the microquasar GRS 1915+105, for instance, it has been suggested (see Tagger et al. 2004) that the long term evolution of the field configuration could be of the order of one to a few years so that half of the time the field fluxes in the inner disk edge and in the $\mathrm{BH}$ would be antiparallel and the rest of the time parallel. Tchekhovskov et al. (2014) suggest that the time scales might be possibly regulated by the accretion time-scale at the outer radii of the disk $\left(t_{a c c}=\alpha^{-1}(H / r)^{-2} \Omega_{K}^{-1}\right.$, where $\Omega_{K}=\left(G M / r^{3}\right)^{1 / 2}$, with $\alpha$ being the disk viscosity parameter, $H$ its height and $r$ the radial distance) which may imply much faster time scales. Numerical simulations of MRI-driven MHD turbulence, which involves part of a mean field dynamo growth, show that generally $\sim 10$ orbital periods are required to reach saturation (Stone et al. 1996), but the building of the large scale poloidal component may take longer and thus the time for its inversion in the disk. Dexter et al. (2014) performed 3D MHD long term simulations letting material to be accreted with a magnetic field with opposite polarity with respect to that attached to the $\mathrm{BH}$ and detect a field inversion in the later after $t \sim 2 \times 10^{4} R_{S} / c$ (where $R_{S}$ is the Schwarzschield radius), which also indicates a very fast process. All these processes are usually connected with different variability phenomena that 
is detected in $\mathrm{BH}$ sources and span a large interval of time scales. The determination of a precise characteristic time scale at which the system may reach exactly the configuration as idealized in Figure 1 is out of the scope of the present work. Nevertheless, the recent observations of a dynamically important magnetic field near the Galactic Centre black hole (see, e.g., Zamaninasab et al. 2014), as well as relativistic numerical simulations of accretion disk-BH magnetosphere interactions as described above (see McKinney et al. 2012; Dexter et al. 2014) indicate that this is a quite possible configuration in the surrounds of BHs.

The poloidal magnetic field lines built in the corona summed to the disk differential rotation give rise to a wind that removes angular momentum from the system leading to an increase in the accretion rate and thus an increase in the ram pressure of the accreting material. This will further accumulate the magnetic lines in the inner disk coronal region pressing them against the lines anchored in the $\mathrm{BH}$ horizon and facilitating the production of a fast magnetic reconnection event (see GL05 and the neutral zone in Figure 11). As shown in GL05 and GPK10, a fast magnetic reconnection event may release substantial magnetic power. In order to evaluate this amount of magnetic power we need first to characterize the coronal parameters in the inner disk region.

We consider here a strongly magnetized fluid in the surrounds of the $\mathrm{BH}$ for which the condition $R_{i}<L_{m f p}<L$ is fulfilled (where $L$ characterizes the large scale dimension of the system, $L_{m f p} \sim 1.8 \times 10^{4} n^{-1} T^{2} \mathrm{~cm}$ is the ion mean free path for Coulomb collisions, and $R_{i} \sim 2.1 \times 10^{9}(E / B) \mathrm{cm}$ is the ion Larmor radius; see more below). For such scales a weakly collisional or effectively collisional MHD description is more than appropriate (e.g., Kulsrud 1983).

It should be noticed also that we consider a nearly non-relativistic MHD approach for describing the coronal region around the $\mathrm{BH}$ (see also, e.g., Liu et al. 2003). The ion/electron temperatures are smaller than or equal to $\sim 10^{9} \mathrm{~K}$, which makes the fluid approximately nonrelativistic and reasonably well described by the standard equations below. Nevertheless, with regard to reconnection, the fact that the Alfvén speed $v_{A}$ may become comparable to the light speed for the conditions analysed here may imply that eventually fast reconnection becomes nearly relativistic. Based on studies performed mostly for collisionless reconnection (see, e.g., the reviews of Uzdenskv 2011; Lvutikov \& Lazarian 2013; de Gouveia Dal Pino et al. 2014, and references therein), it has been found that the behaviour of slow and fast reconnection in relativistic regimes is compatible with that of non-relativistic reconnection. There is also a recent work by Cho \& Lazarian (2014) where it is demonstrated that relativistic collisional MHD turbulence behaves as in the non-relativistic case. This indicates that collisional turbulent fast reconnection theory (as described, e.g., in the LV99 model, see below) can be directly applicable to the relativistic case (see also Lyutikov \& Lazarian 2013) and we will adopt here this approach.

As remarked, we employ below the standard optically thick, geometrically thin accretion disk model (Shakura \& Sunyaev 1973). In GL05 and GPK10 works, the inner radius of the accretion disk $\left(R_{X}\right)$ was taken at the last stable orbit around the $\mathrm{BH}\left(3 R_{S}\right.$, where $R_{S}=$ $2 G M / c^{2}=2.96 \times 10^{5} M / M_{\odot} \mathrm{cm}$ is the Schwartzschild radius). Although physically possible, this condition may lead to a singularity in the Shakura-Sunyaev disk solutions and therefore, we presently adopt an inner radius $R_{X}=6 R_{S}$, which does not affect much the earlier results numerically but avoids the singularity. For a $\mathrm{BH}$ with stellar mass $M=14 M_{\odot}$ (which is suitable for microquasars) this gives $R_{X}=6 R_{S} \simeq 2.48 \times 10^{7} \mathrm{~cm}$. Besides, this condition ensures that $R_{X}$ and all characteristic large scales of the system will satisfy the condition for employment of the nearly collisional MHD fluid approximation (namely, $R_{i}<<L_{m f p} \lesssim R_{X}$ ).

In order to determine the magnetic field intensity in the inner region immediately before an event of violent magnetic reconnection, we assume the equilibrium between the disk ram pressure and the magnetic pressure of the BH magnetosphere. As in GPK10, we approach the radial accretion velocity by the free fall velocity and also assume that the intensity of the field that was dragged by the disk and anchored into the $\mathrm{BH}$ horizon neighbourhood is of the order of the inner disk magnetic field intensity (see Macdonald et al. 1986, and GL05), which gives

$$
\frac{\dot{M}}{4 \pi R^{2}}\left(\frac{2 G M}{R}\right)^{\frac{1}{2}} \sim \frac{B_{d}^{2}}{8 \pi}
$$

o16

$$
B_{d} \simeq 9.96 \times 10^{8} r_{X}^{-\frac{5}{4}} \dot{m}^{\frac{1}{2}} m^{-\frac{1}{2}} G
$$

where $r_{X}=R_{X} / R_{S}$ is the inner radius of the accretion disk in $R_{S}$ units, $\dot{m}=\dot{M} / \dot{M}_{E d d}$ is the mass accretion rate in $\dot{M}_{E d d}$ units (which corresponds to the Eddington mass accretion rate $\dot{M}_{E d d}=1.45 \times 10^{18} \mathrm{mg} / \mathrm{s}$ ), and $\mathrm{m}=$ $M / M_{\odot}$ is the $\mathrm{BH}$ mass in solar mass units.

To quantify the parameters of the corona right above the inner disk region, as in GL05 and GPK10 we employ the model of Liu et al. (2002):

$$
\begin{gathered}
T_{c} \simeq 1.74 \times 10^{6} \Gamma^{\frac{1}{4}} B_{d}^{\frac{3}{4}} L^{\frac{1}{8}} U_{\text {rad }}^{-\frac{1}{4}} K, \\
n_{c} \simeq 9.64 \times 10^{17} \Gamma^{\frac{1}{2}} B_{d}^{\frac{3}{2}} L^{-\frac{3}{4}} U_{\text {rad }}^{-\frac{1}{2}} \mathrm{~cm}^{-3},
\end{gathered}
$$

where $T_{c}$ and $n_{c}$ are the coronal temperature and density, respectively, $L$ is the size of a coronal magnetic flux tube (which will also characterize the height of the corona), $U_{r a d}$ is the disk soft radiation energy density. In GL05 and GPK10, to evaluate this quantity we neglected for simplicity the effects of disk opacity. Here, we give $U_{\text {rad }}$ in terms of the effective temperature at the disk surface (e.g., Frank et al. 2002; Liu et al. 2003):

$$
U_{\text {rad }}=a T_{e f f}^{4}=\frac{4}{c} \frac{3 G M \dot{M} q^{4}}{8 \pi R_{X}^{3}} .
$$

6 We note that in GPK10, eq. (1) was parametrized in terms of $\beta$, namely the ratio between the total disk pressure (gas + radiation pressure) and the magnetic pressure, rather than in terms of $\dot{m}$. In the case of a radiation pressure dominated disk (Shakura \& Sunvaev 1973), both parameters are related through the equation: $\beta \simeq 0.12 \alpha^{-1} r_{X} \dot{m}^{-1}$. 
where $q=\left[1-\left(3 R_{S} / R_{X}\right)^{1 / 2}\right]^{1 / 4}$.

Using equations (2) and (5), the coronal parameters can be rewritten as

$$
\begin{gathered}
T_{c}=2.73 \times 10^{9} \Gamma^{\frac{1}{4}} r_{X}^{-\frac{3}{16}} l^{\frac{1}{8}} q^{-1} \dot{m}^{\frac{1}{8}} \quad K, \\
n_{c} \simeq 8.02 \times 10^{18} \Gamma^{\frac{1}{2}} r_{X}^{-\frac{3}{8}} l^{-\frac{3}{4}} q^{-2} \dot{m}^{\frac{1}{4}} m^{-1} \mathrm{~cm}^{-3},
\end{gathered}
$$

where $l=L / R_{S}$. Also, instead of employing $v_{A} \simeq c$ as in GL05 and GPK10, we have replaced $v_{A}$ by its relativistic form $v_{A}=\Gamma v_{A 0}$, with $v_{A 0}=B /\left(4 \pi \mu m_{H} n_{c}\right)^{1 / 2}$, $m_{H}=m_{p}$ is the proton rest mass, $\mu \sim 0.6$, and $\Gamma=\left[1+\left(\frac{v_{A 0}}{c}\right)^{2}\right]^{-1 / 2}$ (e.g., Somov 2012)].

As described in GL05 and GPK10, the rate of magnetic energy that can be extracted from the reconnection (neutral) zone in the corona (above and below the disk in Figure 10 through reconnection is $\dot{W}_{B}=$ $\left(B^{2} / 8 \pi\right) \xi v_{A}\left(4 \pi R_{X} L_{X}\right)$, where $\xi=v_{r e c} / v_{A}$ is the magnetic reconnection rate, $v_{r e c}$ is the reconnection velocity, and $L_{X}$ is the length of the reconnection region. Mass flux conservation and Figure 1 imply that $\Delta R_{X} / L_{X}=$ $v_{r e c} / v_{A}$. Therefore,

$$
\dot{W}_{B}=\frac{B^{2}}{8 \pi} v_{A}\left(4 \pi R_{X} \Delta R_{X}\right),
$$

where $B$ is the coronal magnetic field in the reconnection zone which is of the order of $B_{d}$, and $\Delta R_{X}$ is the width of the current sheet. Its estimate depends on the fast reconnection model adopted.

\subsection{Fast Reconnection driven by anomalous resistivity}

In GL05 and GPK10 we assumed that fast reconnection is driven by anomalous resistivity (see Parker 1979; Biskamp et al. 1997, LV99). This is based on the onset of current driven instabilities, that can enhance the microscopic Ohmic resistivity and speed up reconnection (e.g. Papadopoulos 1977; Parker 1979; Biskamp et al. 1997). It gives rates which are much faster than the standard Sweet-Parker slow reconnection (which is driven by Ohmic resistivity) and may be naturally present in regions of strong magnetic fields, like those around the $\mathrm{BH}$. When the electron-ion drift velocity exceeds the electron thermal velocity, there can be an electron runaway which causes the formation of electron beams that in turn generate plasma electrostatic waves, giving rise to collective interactions. Electrons are scattered by these fields rather than by individual ions and the classical Spitzer resistivity is replaced by an anomalous resistivity (e.g., Papadopoulos 1977). Following (Parker 1979; Biskamp et al. 1997, LV99, see also GL05), we can estimate the width of the current sheet for anomalous resistivity:

$$
\Delta R_{X}=\frac{c \Delta B}{4 \pi n_{c} Z e v_{t h, c}},
$$

where $\Delta B \simeq 2 B \simeq 2 B_{d}$, and $v_{t h, c}=\left(k T_{c} / m_{p}\right)^{1 / 2}$ is the thermal velocity of the ions of charge $Z e$ in the corona. From equations (2), (6) and (7), we obtain:

\footnotetext{
${ }^{7}$ We note that for the parametric space considered here, $0.36 \lesssim$ $\Gamma \lesssim 0.99$ is obtained numerically from the solution of the equation $v_{A 0} \simeq 9.78 \times 10^{10} \Gamma^{-\frac{1}{4}} r_{X}^{-\frac{17}{16}} l^{\frac{3}{8}} q \dot{m}^{\frac{3}{8}}$.
}

$$
\Delta R_{X} \simeq 2.02 \Gamma^{-\frac{5}{8}} r_{X}^{-\frac{25}{32}} l^{\frac{11}{16}} q^{\frac{5}{2}} \dot{m}^{\frac{3}{16}} m^{\frac{1}{2}} \mathrm{~cm} .
$$

Under these conditions, the magnetic energy power released during violent fast magnetic reconnection in the surrounds of a $\mathrm{BH}$ is approximately given by

$$
\dot{W}_{B} \simeq 2.89 \times 10^{34} \Gamma^{\frac{1}{8}} r_{X}^{-\frac{107}{32}} l^{\frac{17}{16}} q^{\frac{7}{2}} \dot{m}^{\frac{25}{16}} m^{\frac{1}{2}} \mathrm{erg} / \mathrm{s} .
$$

We note that with the adopted normalization, $\Gamma, r_{X}$, $l, q, \dot{m}$, and $m$ are dimensionless parameters.

\subsection{Fast magnetic reconnection driven by turbulence}

In the previous section, we derived the magnetic power released by fast magnetic reconnection due to anomalous resistivity (see Parker 1979; Biskamp et al. 1997). Eq. (10) shows that the resulting thickness of the reconnection region is very small compared to the large scales of the system. Though still much larger than the ion Larmor radius $\left(R_{i} \simeq 6.4 \times\right.$ $\left.10^{-5} \Gamma^{1 / 8} r_{X}^{37 / 32} l^{1 / 16} q^{-1 / 2} m^{1 / 2} \dot{m}^{-7 / 16} \mathrm{~cm}\right)$, it indicates that anomalous resistivity prevails only at the small scales of the system.

On the other hand, the systems we are dealing with (microquasars and AGNs), as most astrophysical systems, have very large Reynolds number, $R_{e} \sim L V / \nu \sim 10^{20}-10^{28}$, where $V$ is a characteristic fluid velocity, $\nu$ is the viscosity which for a magnetically dominated fluid is mainly normal to the magnetic field and is given by $\nu_{\perp} \simeq 1.7 \times$ $10^{-2} n \ln \Lambda T^{-1 / 2} B^{-2} \mathrm{~cm}^{2} \mathrm{~s}^{-1}$, where $\ln \Lambda$ is the Coulomb logarithm, $\Lambda=3 / 2 e^{3}\left(k^{3} T^{3} / \pi n\right)^{-1 / 2} \min [1,(4.2 \times$ $\left.10^{5} / T\right)^{1 / 2}$ ] (see Zhang \& Yan 2011; Spitzer 1962). Similarly, the magnetic Reynolds number $R_{m}=L V / \eta \sim$ $10^{18}-10^{24}$, where $\eta$ is the magnetic resistivity which in the regime of strong magnetic fields is given by $\eta_{\perp} \simeq$ $1.3 \times 10^{13} Z \ln \Lambda T^{-3 / 2} \mathrm{~cm}^{2} s^{1}$ (Spitzer 1962). These large Reynolds numbers imply that the fluid and the magnetic fields can be highly distorted and turbulent if there is turbulence driving. In other words, the growth of any instability as for instance, the current driven instabilities mentioned above (like, e.g., the Buneman instability which occurs when $T_{e} / T_{i} \sim 1$, where $T_{e}$ and $T_{i}$ are the electron and ion temperatures, respectively, Papadopoulos (1977) can naturally drive turbulence with characteristic velocities around the particles thermal speeds. Also, the occurrence of continuous magnetic reconnection with the ejection of plasmoids during the building of the corona itself in the surrounds of the BH (Liu et al. 2002, 2003) will contribute to the onset of turbulence. Numerical simulations of coronal disk accretion also indicate the formation of turbulent flow in the surrounds of the $\mathrm{BH}$ that may be triggered, e.g., by magnetorotational instability (see e.g, Tchekhovskoy et al. 2011; McKinney et al. 2012; Dexter et al. 2014).

Turbulence is known to speed up the reconnection. In this case, we can examine an alternative model of fast

${ }^{8}$ We note that we have also explored the effects of general relativity in the gravitational potential in the surrounds of the $\mathrm{BH}$ and recalculated the magnetic reconnection power in the inner disk region considering a pseudo-Newtonian potential and found that for the scales considered here with $R_{X} \simeq 6 R_{S}$ these are negligible. 
reconnection driven by turbulence. We here adopt the turbulent collisional fast reconnection model introduced by LV99.

According to LV99 theory, the presence of even weak turbulence in a collisional flow causes the wandering of the magnetic field lines (facilitated by Richardson diffusion, see below) which allows for simultaneous events of reconnection to occur in small patches making reconnection naturally fast and independent of the Ohmic resistivity. In other words, the spontaneous stochasticity introduced by the weak turbulence in the mean field causes the diffusion of the magnetic field lines, which is a macroscopic process independent of microscopic resistivity. The reconnection over small patches of magnetic field determines the local reconnection rate. The global reconnection rate is substantially larger as many independent patches reconnect simultaneously. In other words, the LV99 model predicts that the small scale events happen at a slow (Sweet-Parker) rate, but the net effect of several simultaneous events makes reconnection fast. This theory has been thoroughly discussed in the literature in many papers and reviews (see, e.g., Eyink et al. 2011, 2013; Lazarian et al. 2012, 2015) and successfully tested by means of 3D MHD simulations (Kowal et al. 2009, 2012; Xu \& Yan 2013). by:

In the LV99 theory, the reconnection velocity is given

$$
v_{r e c} \simeq v_{A} \min \left[\frac{L_{i n j}}{L_{X}}, \frac{L_{X}}{L_{i n j}}\right]^{\frac{1}{2}} M_{A}^{2},
$$

where $L_{X}$ is the extension of the reconnection zone as in Figure 1] $M_{A}=v_{i n j} / v_{A}$ is the Alfvénic Mach number of the turbulence, $v_{i n j}$ and $L_{i n j}$ are the turbulent velocity and length, respectively, at the injection scale. The three-dimensional numerical MHD simulations by Kowal et al. (2009, 2012) indicated a slightly distinct dependence in the power-law index in the equation above, i.e., $v_{r e c} \propto L_{i n j}^{1 / 4}$.

The equation above was also derived using the wellknown concept of Richardson diffusion (Eyink et al. 2011). From the theoretical perspective this new derivation avoids the more complex considerations of the cascade of reconnection events that were presented in LV99 to justify the model. These authors have demonstrated that the LV99 model is connected to the "spontaneous stochasticity" of the magnetic field in turbulent fluids (see also Eyink et al. 2011, where numerical simulations demonstrate this diffusion process as well).

Mass conservation $\left(\Delta R_{X} / L_{X}=v_{r e c} / v_{A}\right)$ and equation (12) imply that

$$
\Delta R_{X}=L_{X} \min \left[\frac{L_{i n j}}{L_{X}}, \frac{L_{X}}{L_{i n j}}\right]^{\frac{1}{2}} M_{A}^{2} .
$$

We may employ this relation to compute the magnetic reconnection power from eq. (8). Considering the discussion above, we may assume that the injection velocity of the turbulence is of the order of the coronal gas sound speed and the injection scale of the turbulence is of the order of the size of the reconnection zone, i.e., $L_{i n j} \simeq L_{X}$, which in turn may be set as a free parameter in the model being smaller than the coronal loop size, i.e., $L_{X} \leq L$.
With these assumptions, we may rewrite the equation above as

$$
\Delta R_{X} \simeq 2.34 \times 10^{4} \Gamma^{-\frac{5}{16}} r_{X}^{\frac{31}{64}} l^{-\frac{5}{32}} l_{X} q^{-\frac{3}{4}} \dot{m}^{-\frac{5}{32}} \mathrm{~m} \mathrm{~cm},
$$

where $l_{X}=L_{X} / R_{S}$.

This results a magnetic reconnection width which is much larger than in the case of anomalous resistivity (eq[10) indicating that reconnection driven by turbulence can be much more efficient to drive fast reconnection. As a matter of fact, observations of solar flares indicate fast reconnection rates up to $v_{r e c} / v_{A} \sim 0.1$ (Takasaki et al. 2004), while numerical simulations of turbulent fast reconnection also result values up to $v_{r e c} / v_{A} \sim 0.1$ depending on the turbulent injection power $\left(P \propto v_{i n j}^{2}\right)$. Here, because of the lack of knowledge of the physical details of the injection and power scales of the turbulence, we adopt the analytical relation above for $\Delta R_{X}$.

The fact that turbulence results a much larger reconnection rate than anomalous resistivity is actually not a surprise and comes directly from the different nature of both processes. As stressed before, anomalous resistivity acts only at small scales resulting a much smaller reconnection rate, while collisional turbulence acts on the large scales of the fluid, as we can see, for instance, by comparing the equation above with eq. (10). Kowal et al. (2009) have also compared the two processes employing 3D MHD numerical simulations and found that the introduction of anomalous resistivity is unable to affect the rate of reconnection due to weak turbulence. This supports the notion that in the presence of turbulence and magnetic field stochasticity induced by it, plasma kinetic effects do not seem dominant in the determination of the global reconnection speed.

This point can be better understood considering the condition for having turbulent collisional reconnection according to the LV99 theory. This requires the thickness of the reconnection region (eq14) to be larger than the ion Larmor radius, $\Delta R_{X}>R_{i}$ (Eyink et al. 2011). We find that this condition is satisfied in all the fiducial parametric space investigated here for the physical conditions around the BHs. On the opposite situation, collisionless kinetic effects would prevail to induce fast reconnection.

The result above also suggests that anomalous resistivity may be an important process in the beginning of the reconnection process, but once turbulence is developed in the system, then fast reconnection induced by turbulence will be the dominant process.

Considering the equations (8) and (14), we obtain that the magnetic reconnection power released by turbulent fast reconnection in the surrounds of the $\mathrm{BH}$ is given by

$$
\dot{W}_{B} \simeq 1.66 \times 10^{35} \Gamma^{-\frac{1}{2}} r_{X}^{-\frac{5}{8}} l^{-\frac{1}{4}} l_{X} q^{-2} \dot{m}^{\frac{3}{4}} \mathrm{~m} \mathrm{erg} / \mathrm{s},
$$

which obviously results a larger value than in the case of fast reconnection driven by anomalous resistivity (eq 11).

Figure 2 compares the two values derived for the magnetic reconnection power (equations 11 and 15) as a function of the mass of the central source for a suitable choice of parameters: $R_{X}=6 R_{S} ; 1 \leq m \leq 10^{10}$ (in $M_{\odot}$ units) to spam masses from microquasars to AGNs; and $0.05 \leq \dot{m} \leq 1$ (in $\dot{M}_{E d d}$ units) for the mass accretion 


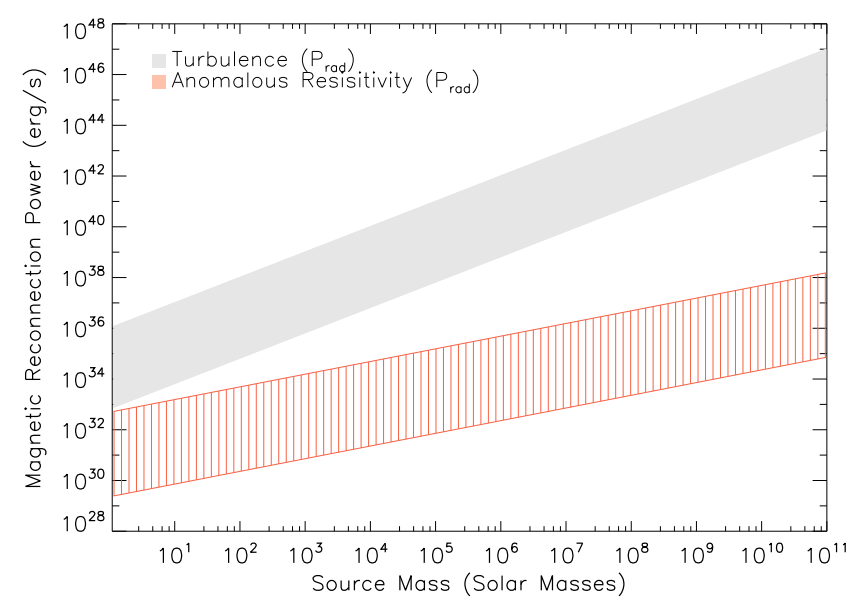

Figure 2. Magnetic power $\left(\dot{W}_{B}\right)$ released by fast reconnection driven by anomalous resistivity (in red) and by turbulence (in light gray) as a function of the $\mathrm{BH}$ mass. The parametric space spans $0.05 \leq \dot{m} \leq 1 ; 1 \leq l \lesssim 18$; and $0.06 l \lesssim l_{X} \leq l$ (assuming $R_{X}=$ $\left.6 R_{S}\right)$.

rate. Also, in order to ensure near collisionality in the flow and the validity of the equations above in the parametric space, we have constrained the lower bound of the characteristic scales of the system to be larger than the ion mean free path. Specifically, we have imposed $l_{m f p} \lesssim l_{X} \leq l$, where $l_{m f p} \simeq 5.70 \times 10^{-2} l \mathrm{~cm}$ is the mean free path in $R_{S}$ units. This gives $1 \leq l \lesssim 18$ (in $R_{S}$ units) for the length of the magnetic loop (or the height of the corona) and $0.06 l \lesssim l_{X} \leq l$ (in $R_{S}$ units). The upper limit of $l$ has been obtained from the condition $l_{m f p} \lesssim 1$.

While in the anomalous resistivity case $\dot{W}_{B}$ has a dependence with the source mass given by $\dot{W}_{B} \propto m^{0.5}$ in the turbulent reconnection case this dependence is steeper $\dot{W}_{B} \propto m$ as evidenced in the Figure 2. We will see in Section 3 that this has important observational consequences for microquasars and AGNs.

\subsection{Radiation-pressure dominated versus gas pressure-dominated accretion disks}

The values of accretion rate employed in Figure 2 are more suitable for a corona connected to a radiationpressure dominated disk. We may also evaluate $\dot{W}_{B}$ from equations (11) and (15) when considering a gas-pressure dominated disk. In this case, the accretion rates cannot be as large as those considered in the radiation regime in Figure 2]

In a gas-pressure dominated regime the disk pressure is given by Shakura \& Sunvaev (1973):

$$
P_{g a s} \simeq 4.11 \times 10^{18} \alpha^{-\frac{9}{10}} r_{X}^{-\frac{21}{8}} q^{\frac{17}{5}} \dot{m}^{\frac{17}{20}} m^{-\frac{9}{20}} \quad \mathrm{dyn} / \mathrm{cm}^{2},
$$

while for a radiation pressure dominated regime the disk pressure is:

$$
P_{r a d} \simeq 4.78 \times 10^{15} \alpha^{-1} r_{X}^{-\frac{3}{2}} m^{-1} \mathrm{dyn} / \mathrm{cm}^{2},
$$

where $\alpha$ is the disk viscosity parameter.

We can compare the two equations above in order to obtain the range of accretion rates which are suitable for each regime. Figure 8 in Appendix $\mathrm{A}$ depicts the ratio between these two pressures as a function of the mass of the sources. We see that for $0.05 \leq \alpha \leq 0.5$ (see King et al. 2007), considering the whole range of masses, $P_{\text {rad }} / P_{\text {gas }}<1$ (gas-pressure dominated regime) for $\dot{m} \leq 5 \times 10^{-4}$, and $P_{\text {rad }} / P_{\text {gas }}>1$ (radiation-pressure dominated regime) for $\dot{m}>5 \times 10^{-2}$. In the next sections, we will adopt these ranges of accretion rates in the computation of the magnetic reconnection power for each accretion disk regime.

\section{COMPARISON OF $\dot{W}_{B}$ WITH THE OBSERVED CORE RADIO AND GAMMA EMISSION OF MICROQUASARS} AND AGNS

In the previous section, we evaluated the magnetic reconnection power produced by fast reconnection in the surrounds of a $\mathrm{BH}$, considering two different mechanisms to induce it, anomalous resistivity and turbulence. The second one was found to be much more efficient. It is out of the scope of the present work to predict what amount of this magnetic power goes to accelerate particles, but as stressed in section 11 (also in GL05 and GPK10), we may expect that a substantial fraction of it will produce high-speed electrons which will spew outward (Kowal et al. 2011, 2012) and produce relativistic ejecta and Synchrotron radio emission. In this section, we first compare the calculated fast magnetic reconnection power with the observed radio emission of the nuclear regions of microquasars and AGNs, then we compare this power with the processed VHE emission from these sources.

\subsection{Low-luminosity sources}

Figure 3 depicts in gray color scales the calculated magnetic power released by a fast magnetic reconnection event as a function of the central BH mass induced both by turbulence (eq 15) in radiation and gas-pressure dominated regimes and by anomalous resistivity in a radiation pressure regime (eq11). The continuous line in the figure corresponds to the observed correlation between the $\mathrm{BH}$ mass and the core radio luminosity for a sample of 96 nearby LLAGNs (within distances of $19 \mathrm{Mpc}$ ) found by Nagar et al. (2002) from VLA and VLBA observations. The dashed line was obtained by Nagar et al. (2005) considering a more refined VLBI sample. The dotdashed line corresponds to the observed correlations by Merloni et al. (2003) considering the VLA 5GHz core radio emission of a sample of $\sim 100$ AGNs (most of which with arcsecond resolution) and the radio emission of 8 galactic black holes obtained with the Green-Bank Interferometer. Despite the simplicity of our model, the slope dependence of the magnetic power released by turbulent reconnection with the source mass is very similar to the observed radio luminosity-source mass correlations for these sources. A closer examination of the diagram shows that the predicted intensities of the turbulent driven magnetic reconnection power in the swept parametric space are much larger than the observed radio luminosities, specially in the upper part of the diagram, i.e., in the radiation pressure-dominated case. This is an indication that in general only a small fraction of the magnetic reconnection power would be enough to explain the observed radio emission for most of the sources represented by the correlation lines. The anomalous resis- 


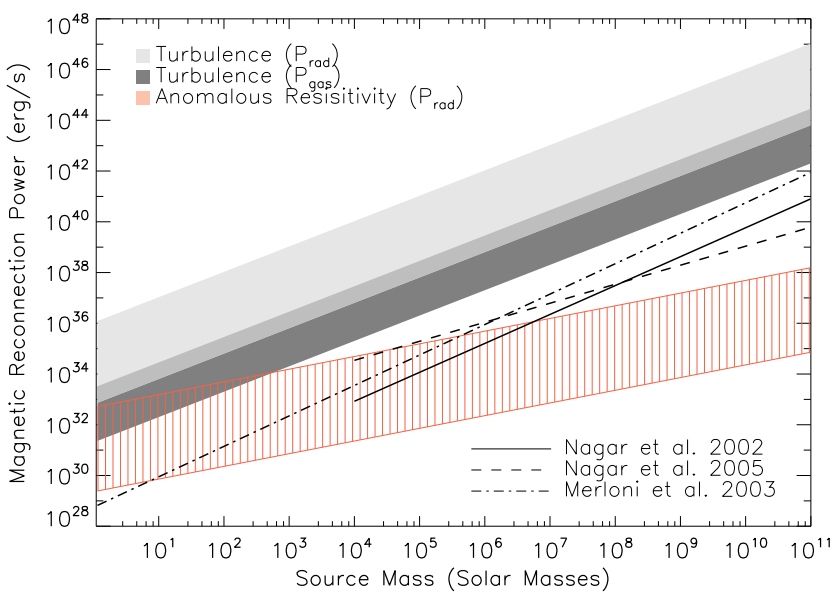

Figure 3. Magnetic power $\left(\dot{W}_{B}\right)$ released by fast reconnection driven by anomalous resistivity (in red) and by turbulence as a function of the $\mathrm{BH}$ mass. The upper part of the diagram of the turbulent driven reconnection power (light gray) corresponds to a radiation-pressure dominated disk with larger accretion rates $(0.05 \leq \dot{m} \leq 1)$, while the lower part of the diagram (dark gray) stands for a gas-pressure dominated disk with $\dot{m} \simeq 5 \times 10^{-4}$ (see appendix $\mathrm{A}$ and Figure 8 for details); and the intermediate gray region is the overlap between both regimes. The diagram of the magnetic reconnection power driven by anomalous resistivity also corresponds to a radiation-pressure dominated disk. The other free parameters in the diagrams span $1 \leq l \lesssim 18$; and $0.06 l \lesssim l_{X} \leq l$ (assuming $R_{X}=6 R_{S}$ ), as in Figure 2 The continuous and dashed lines correspond to the observed correlations between the $\mathrm{BH}$ mass and the core radio luminosity found for LLAGNs by Nagar et al. (2002) and Nagar et al. (2005), respectively; and the dot-dashed line corresponds to observed correlations for AGNs and microquasars by Merloni et al. (2003).

tivity model on the other hand does not match most of the observed correlations for the parametric space considered. In GPK10 work, the comparison of the magnetic reconnection power driven by anomalous resistivity with the radio luminosity of a bunch of microquasars and LLAGNs had revealed a better match between both because in that case, the magnetic reconnection power was computed considering a larger disk radiation field than the present evaluation (eq[5) making the corona hotter (eq 6) and therefore, the magnetic reconnection more efficient. Also, in GPK10 we considered coronal heights up to nearly $1000 R_{S}$ which in that approach made the upper bound of the reconnection power larger than in the present case.

Figure 4 compares the calculated magnetic reconnection power driven by turbulence with the observed core radio luminosities of a large sample of LLAGNs and microquasars, namely 9 microquasars (or galactic black holes - GBHs, see Hannikainen et al. 2001; Merloni et al. 2003), and 233 LLAGNs (including Seyferts and LINERs galaxies, see Merloni et al. 2003; Nagar et al. 2002, 2005; Israel 1998; Kadler et al. 2012). Table 1 lists the physical parameters for this sample. The $\mathrm{BH}$ masses of the sources (also indicated in Table 10 were evaluated from averages taken from different determinations in the literature including kinematic methods using stellar, gas, or maser dynamics (Richstone et al. 1998; Gebhardt et al. 2000; Merloni et al.|2003; Remillard \& McClintock|2006), and the relation between the $\mathrm{BH}$ mass and the dispersion velocity at the center of the host galaxy (Tremaine et al.
2002; Merritt \& Ferrarese 2001) obtained from the HYPERLEDA catalogu母?

The radio emission from the sources in Figure 4 is represented by diamond symbols, with the red color corresponding to LLAGNs (LINERs an Seyfert galaxies) and the green color to microquasars. We also highlighted the location of the observed radio emissions of a few sources that have been extensively explored in multi-wavelength campaigns, i.e., the radio galaxies Cen A, M87, IC 310, and Per A (NGC 1275), and the microquasars Cgy-X1 and Cgy-X3. Figure 4 confirms the trend of the previous Figure 3 indicating that the magnetic reconnection power extracted from reconnection of the magnetic lines in the inner coronal region around the $\mathrm{BHs}$ of microquasars and LLAGNs could be enough to explain the core Synchrotron radio emission from them, as suggested already by GPK10 for a much smaller sample of sources (and considering a fast magnetic reconnection model driven by anomalous viscosity). Actually, the results indicate that for most of the sources only a small fraction of the magnetic reconnection power would be sufficient to accelerate the electrons responsible for the radio Synchrotron emission.

Laboratory experiments of magnetic reconnection Yamada et al. 2014) and solar flare observations (Lin \& Hudson 1971) indicate that 50-60\% of the magnetic power released by reconnection can go into particle acceleration. Figure 3 indicates that there is in general much more power available to accelerate particles than that spent in the radio Synchrotron emission. As discussed in Section 1. accelerated relativistic electrons along with accelerated protons will also cool via other mechanisms that will lead to $\mathrm{HE}$ and VHE emission. These processes include inverse Compton (IC) relativistic electron interactions with the surrounding photon field, or Synchrotron-self Compton (SSC) interactions with the Synchrotron photons they produce, or proton interactions with surrounding protons (via p-p interactions) and photons (via p-photon interactions) (e.g., Romero et al. 2003; Khiali et al. 2015; Aleksić et al. 2010b; Abdo et al. $2009 \mathrm{a}, \mathrm{b}$, and references therein). For this reason in Figure 5 we have also plotted the observed gamma-ray luminosities (see Table 1) which is available for only a subsample of 23 sources of those depicted in Figure 4 . For most of the Seyferts galaxies of this subsample, the figure shows only upper limits of the gamma-ray luminosity in the $\mathrm{GeV}$ band (obtained with $95 \%$ confidence level by Ackermann et al. (2012) with Fermi-LAT). These data are represented by circle symbols in Figure 5 , and Table 1 provides more information on the gamma-ray luminosity for these sources. We included also the observed gamma-ray luminosities of the four radio galaxies highlighted in the figure (Abdo et al. 2009b, 2010; Aleksić et al. 2014a, b), as well as of the microquasars Cgy-X1 (Albert et al. 2007; Malyshev et al. 2013) and Cgy-X3 (Piano et al. 2012). In these cases, there is data available from $\mathrm{MeV} / \mathrm{GeV}$ to $\mathrm{TeV}$ bands and we represented the whole luminosity range (from the maximum to the minimum observed values) with circles linked by vertical lines that also extend to the radio emission of each of these sources (see also Table 1).

Figure 5 shows that the magnetic reconnection power

9 http://www-obs.univ-lyon1.fr/hypercat/ 


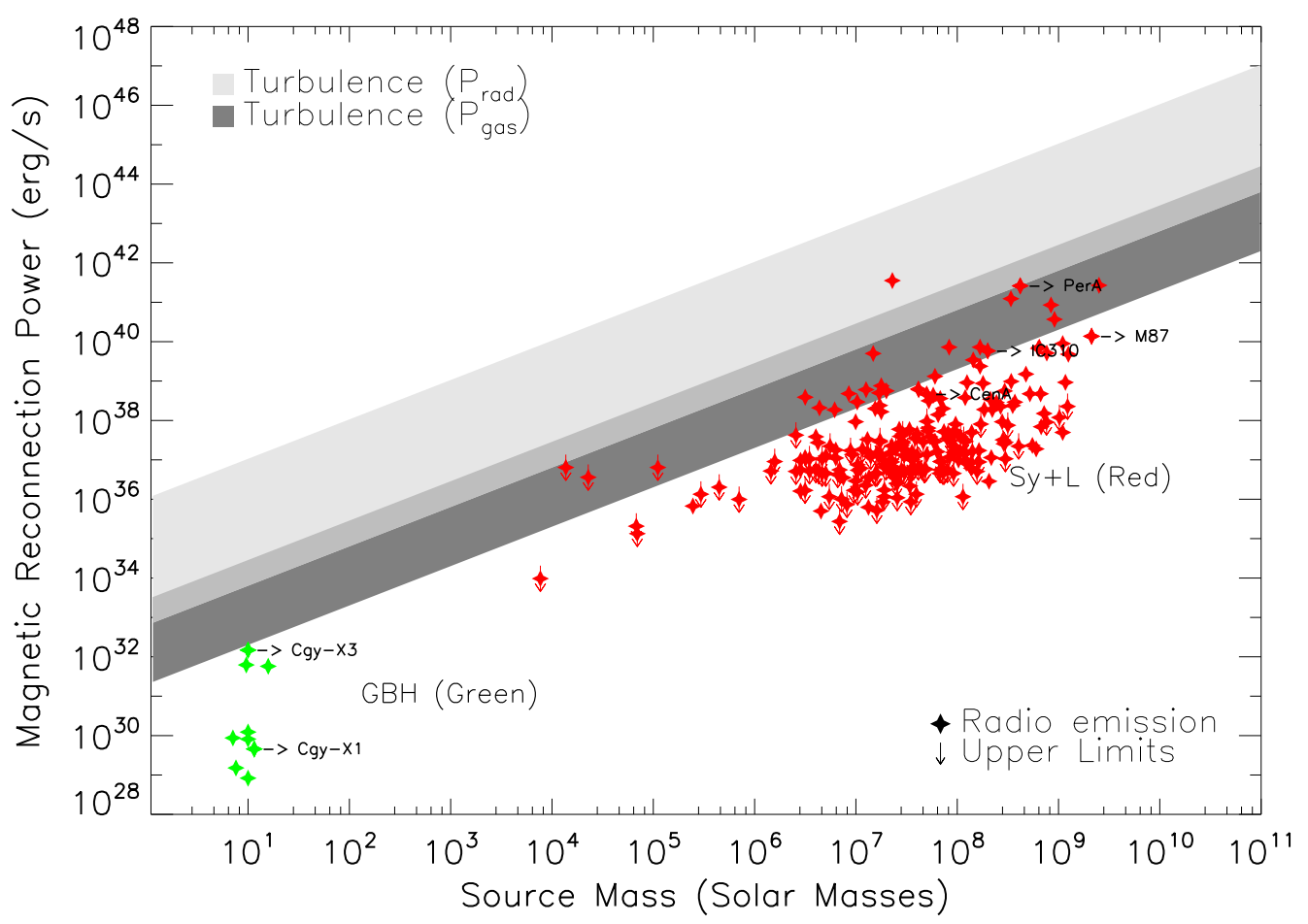

Figure 4. Turbulent driven magnetic reconnection power $\dot{W}_{B}$ (eq15) against BH source mass, as in Figure 3 compared to the observed core radio emission of 233 LLAGNs and 9 microquasars (GBHs). The parametric space used in the calculation of $\dot{W}_{B}$ is the same as in Figure 3 The green diamonds give the observed core radio luminosities for microquasars, the red diamonds the core radio luminosities of LLAGNs (LINERS and Seyfert), and the arrows indicate upper limits data. The position of the observed emission for a few sources is highlighted with black arrows.

diagram also encompasses the observed gamma-ray emission of the low luminosity sources, though we cannot predict what fraction of this power might be required to produce it. In fact, because the $\mathrm{HE}$ and VHE emission of the sources do not depend only on the energy of the accelerated primary relativistic particles, but also on the surrounding photon and proton density fields of the source, one should not expect that the magnetic reconnection power might also directly probe this emission. If the released $\dot{W}_{B}$ is the responsible for the acceleration of the relativistic particles then, it must be larger than (or at least comparable, depending on the energy transfer efficiency) to the associated electron radio synchrotron radiation, as we find. Nevertheless, a striking feature in Figure 5 is the fact that there is in principle power enough to produce also the gamma-ray emission (of course, with different amounts for different sources) and this emission nearly follows the same trend of the observed radio emission in the diagram, and thus both emissions are correlated and could be possibly produced in the same region in the core, by the same relativistic particle populations, which in turn can be due to the magnetic reconnection mechanism as described.

We note that upper limits of the gamma-ray luminosity of a much larger sample of Seyfert galaxies obtained by Ackermann et al. (2012), but with no counterpart in the radio emission sample of Figure 5] were also found to match and follow the same trend of the other sources represented in the diagram of Figure 5

\subsection{Inclusion of high-luminosity sources}

In the previous section, we discussed the correlation between the magnetic power released by fast magnetic reconnection at the inner corona/accretion disk region, and the observed radio and gamma-ray luminosities of LLAGNs and microquasars. In this section we extend this analysis to a much broader sample that includes blazars and GRBs, i.e., high-luminosity sources.

As stressed in Section 1, in blazars the jet is known to point towards the line of sight screening most of the inner core radiation, but the observed radio emission is often separated in a core (probably produced near the jet basis) and an extended component (e.g., Kharb et al. 2010). Figure 6 and Table 2 present the core (or jet basis) radio emission of these blazars along with the core radio emission of LLAGNs. As in Figure 4. we compare the observed emission of these sources with the calculated turbulent driven magnetic reconnection power. This sample has 32 blazars studied by Nemmen et al. (2012) (whose black hole masses and radio emission were obtained from Vovk \& Neronov (2013) and Kharb et al. (2010), respectively). The dashed vertical bars associated to the blazars emission give the corrected radio luminosity due to Doppler beaming. We performed the same correction as Nemmen et al. (2012) did for the gamma-ray luminosity of these sources 10

10 Nemmen et al. (2012) assumed an isotropic gamma-ray emission and then corrected it by the beaming factor $f_{b}$, i.e., $L_{c o r r}=$ $f_{b} L_{i s o}$, where $L_{c o r r}$ is the corrected luminosity, $L_{i s o}$ is the isotropic luminosity, and $f_{b}=1-\cos \left(1 / \Gamma_{0}\right)$, where $\Gamma_{0}$ is the bulk 


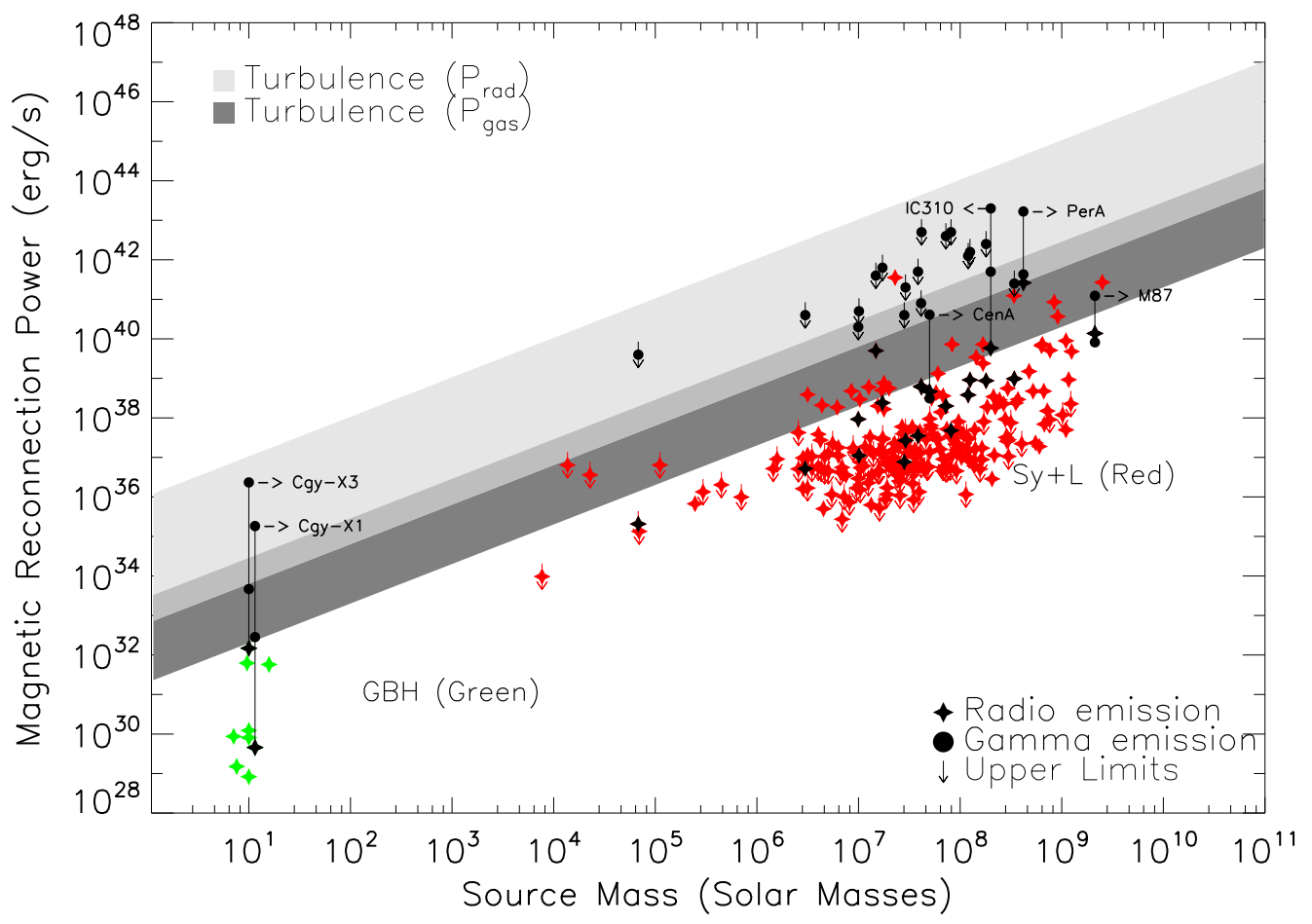

Figure 5. The same as in Figure 4 but now including also the gamma-ray emission of a subsample of LLAGNs, and microquasars for which this emission is available. This emission is represented by black circles. The radio emission of the corresponding sources is also represented by black stars to distinguish them from the rest of the sample. In a few cases for which there is observed gamma-ray luminosity from $\mathrm{MeV} / \mathrm{GeV}$ to $\mathrm{TeV}$ ranges, we plotted the maximum and minimum values linking both circles with a vertical black line that extends down to the radio emission of each source. The arrows associated to some sources indicate that the gamma-ray emission is an upper limit only.

Figure 6] indicates two striking features, with the Doppler boosting correction, most of the blazars radio emission lies in the highest accretion rate part of the $\dot{W}_{B}$ diagram. This is compatible with the notion that this emission is actually produced near the jet launching basis and therefore, could well be triggered by fast magnetic reconnection as in the LLAGNs. On the other hand, in spite of the uncertainties in the determination of the Doppler boosting correction and the poorness of the sample, the blazar emission does not seem to follow the same trend as that of the LLAGNs, specially the highest luminosity ones. This may be an indication that their emission, specially that of the highest luminosity sources, comes mostly from further out in the jet basis, and may be due to another population of relativistic particles, probably produced already at the jet shock, as it is generally expected.

To strengthen the conclusion above, in Figure 7 we have plotted the observed gamma-ray emission for the 32 blazars (Nemmen et al. 2012, see also Table2) along with the observed gamma and radio emission of the LLAGNs. Even with the Doppler correction, we note that most of the blazars gamma-ray emission lies above the $\dot{W}_{B}$ diagram. Besides, as the radio emission, it does not follow the same trend of the LLAGNs.

For comparison, we have also plotted the gamma-ray emission of a sample of 54 GRBs (also obtained from Nemmen et al. 2012, see Table 3). Figure 7 clearly shows

Lorentz factor. that neither the blazars nor GRBs have their gammaray luminosity correlated with $\dot{W}_{B}$. Actually, the trend that links these two classes of sources in the diagram is anti-correlated with the gray zone that characterizes the nuclear magnetic reconnection emission mechanism here discussed. This suggests that the gamma emission in these sources cannot be attributed to this emission process. This result is consistent to what is usually expected in the case of the GRBs, i.e., that their gamma-ray emission does not come from the core or the engine which is totally embedded in the envelope of the progenitor (e.g., Paczyński 1998; MacFadyen \& Woosley 1999). In the case of the blazars, this result also indicates that their nuclear emission is probably obscured by the jet pointing to us and therefore, what we really observe is gamma-ray emission coming predominantly from the optically thin jet.

\section{DISCUSSION AND CONCLUSIONS}

We have here extended the earlier work by GL05 and GPK10, investigating the interactions between the magnetic field lines that arise from the accretion disk and the magnetosphere anchored into the $\mathrm{BH}$ horizon of microquasars and AGNs. We examined the conditions under which fast magnetic reconnection events can occur in this inner region and release enough magnetic power to enable the heating and acceleration of particles to relativistic velocities. 


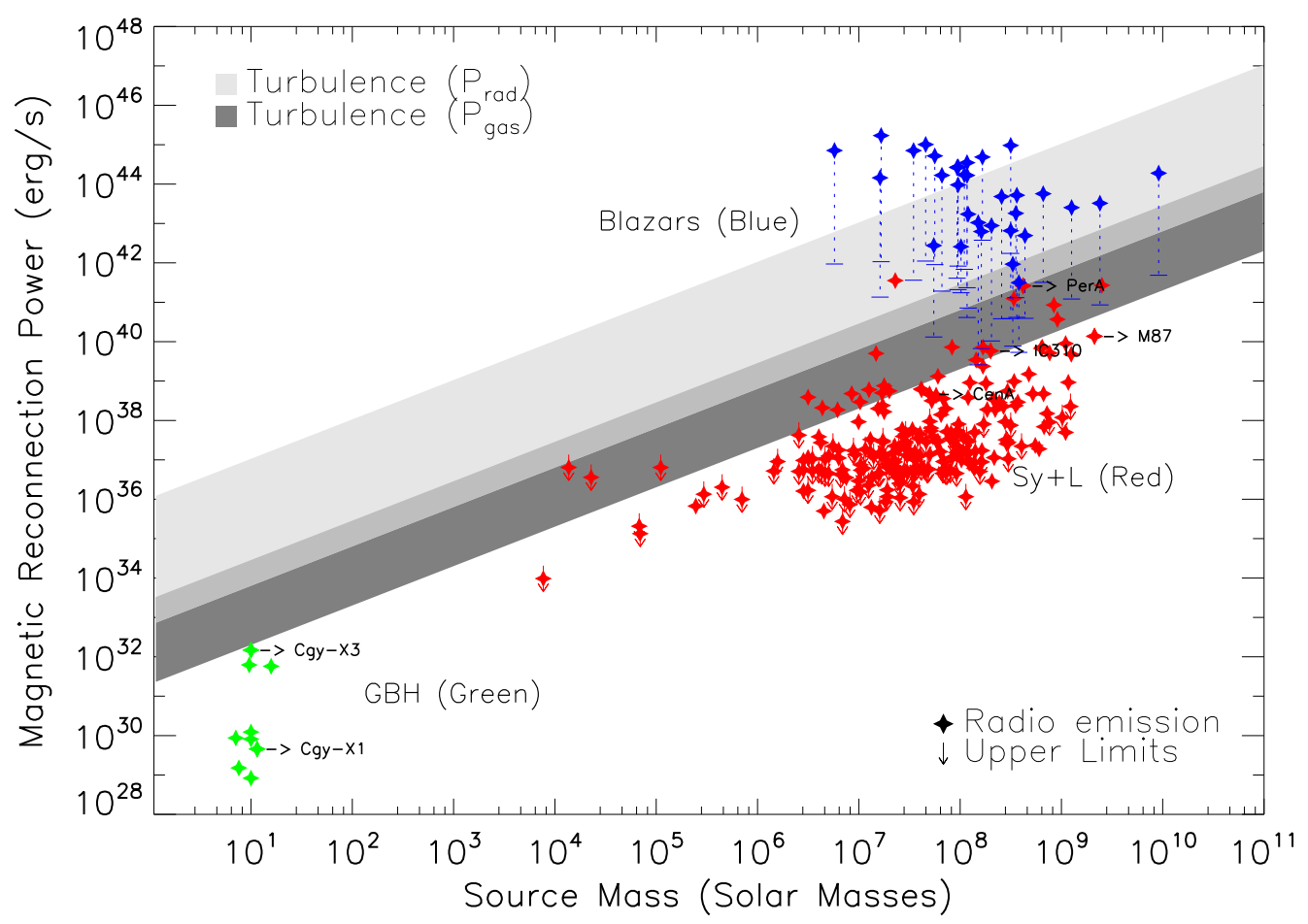

Figure 6. The same as in Figure 4 but now including also the observed radio emission from blazars (represented in blue diamonds). The vertical dashed lines correct the observed emission by the effects of Doppler boosting (see more details in the text).

4.1. Anamalous resistivity versus turbulence induced fast reconnection and other processes

Reviewing the potential mechanism(s) that can excite fast reconnection in this nearly collisional MHD scenario, we compared the earlier investigated process in GL05 and GPK10, namely, anomalous resistivity (Parker 1979; Biskamp et al. 1997; Shav et al. 1998), with reconnection driven by turbulence (Kowal et al. 2009, LV99). We have found that the magnetic power released by turbulent driven fast reconnection (eq 15) is much larger than that obtained by anomalous resistivity (eq[11), as the presence of turbulence naturally enlarges the thickness and the volume of the magnetic reconnection discontinuity (eq14). This result is not a surprise, since anomalous resistivity acts dominantly at small scales resulting a much smaller reconnection rate, while collisional turbulence acts on the large scales of the fluid (see eqs. 10 and 14 that compare the thickness of the reconnection zone for both processes).

Back to 2005, GL05 (see also GPK10) explored only the effects of anomalous resistivity in driving fast reconnection in the surrounds of BHs, because this process was already largely studied, while the LV99 theory was still under testing. Currently, LV99 theory has been thoroughly discussed considering different approaches (e.g., Evink et al. 2011; Lazarian et al. 2012, 2015) and successfully tested by means of 3D MHD simulations (Kowal et al. 2009, 2012; Evink et al. 2013; Xu \& Yan 2013), therefore motivating its examination in the present analysis. Interestingly, we have found that it is able to reproduce much better the observations (see section 3 and below).

The perception that turbulence might affect magnetic reconnection (as in LV99) is not unprecedented (for a review with a comparative analysis of the different models see Kowal et al. 2009; Evink et al. 2011; Lazarian et al. 2015). Several earlier works focussed on the effects of turbulence at microphysical scales (e.g., Speiser 1970; Jacobson \& Moses 1984), but at the MHD large scale level these kinetic effects are not dominant. The closest study to LV99 model was done by Matthaeus \& Lamkin (1985, 1986). These authors explored 2D magnetic reconnection in the presence of turbulence and found a significant enhancement in the reconnection rate. However, they did not derive an analytical prediction for the reconnection speed. Other works have introduced the hyper-resistivity concept and tried to derive fast reconnection rates from turbulence within the context of mean-field resistive MHD (Strauss 1986; Bhattacharjee \& Hameiri 1986; Hameiri \& Bhattachariee 1987; Diamond \& Malkov 2003; Yokoi \& Hoshino 2011; Guo et al. 2012). Though the approach at first level seems interesting, these works have reached different results in the estimates of the reconnection rate and besides, they still lack multidimensional numerical testing.

Several possibilities of fast reconnection in the collisional MHD regime driven by instabilities in the reconnection layer have been also largely discussed (e.g., Loureiro et al. 2009; Bhattacharjee et al. 2009). As remarked in Eyink et al. (2011), these ideas can be traced back to the work of Shibata \& Tanuma (2001) (see also Strauss 1986; Waelbroeck 1989) who suggested that tearing instability may result in fractal reconnection taking place on very small scales. Estimates indicate that laminar current sheets subject to tearing instability have re- 


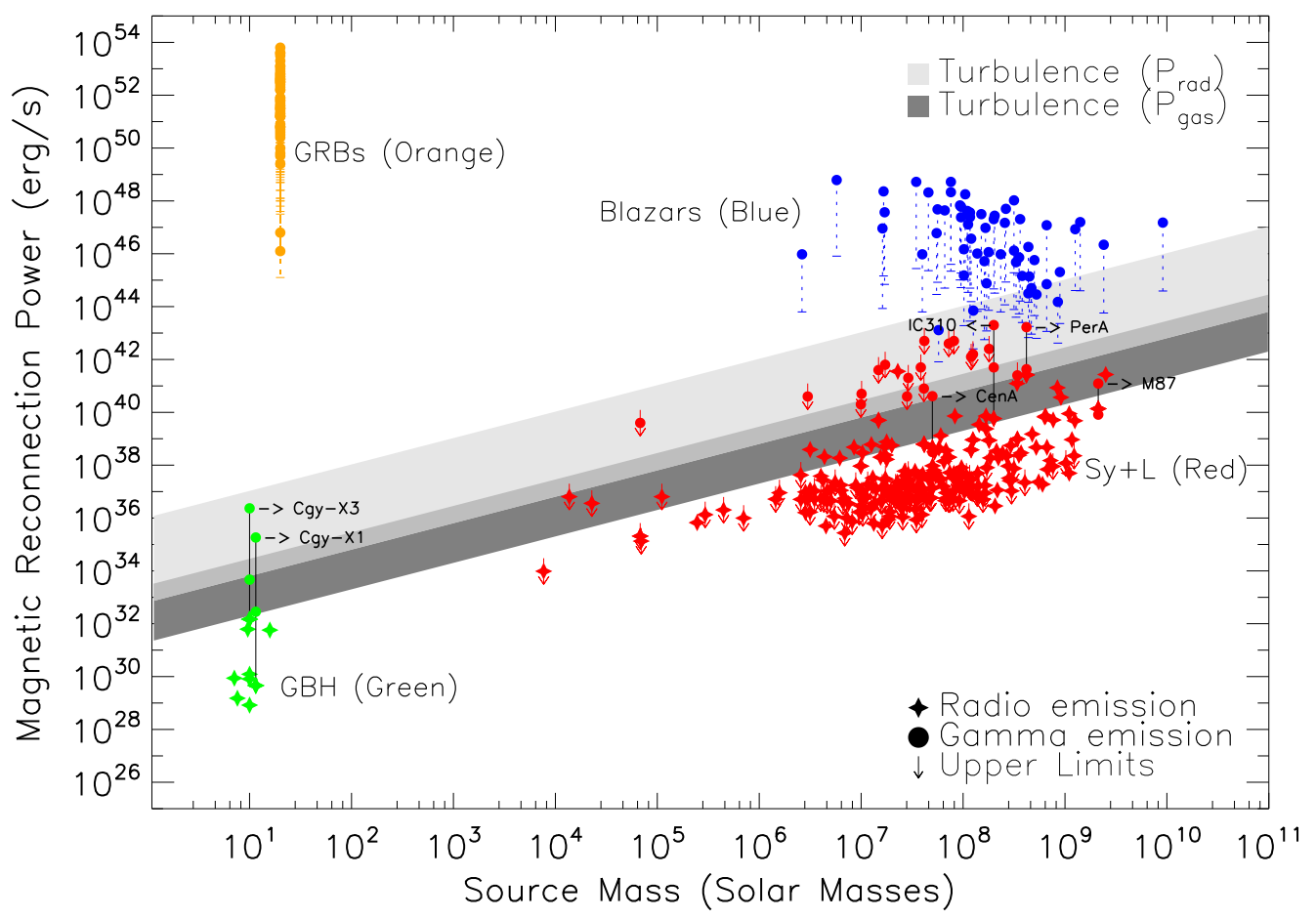

Figure 7. The same as in Figure 5 but now including also the gamma-ray emission from blazars (represented in blue circles) and GRBs (represented in orange circles). The vertical dashed lines correct the observed emission by the effects of Doppler boosting (see more details in the text).

connection rates that are a little faster than the SweetParker, but they enlarge the reconnection layer enabling a wide outflow which will become turbulent in most astrophysical conditions. In this case, the instability can be important for initiating reconnection when the level of pre-existing turbulence is still low, but once turbulence becomes dominant this will dominate reconnection making it very fast. In conclusion, like anomalous resistivity, we expect these instabilities to be important for the onset of reconnection and turbulence, therefore increasing the three-dimensional stochasticity of magnetic field lines and thus initiating large scale fast reconnection, as proposed in LV99.

\subsection{Particle acceleration induced by the magnetic power released by reconnection}

In the present work we focussed on the derivation of the magnetic power released by fast magnetic reconnection and then, arguing that part of this energy would be able to accelerate particles to relativistic velocities, we compared this power with the observed radio and gammaray luminosities of $\mathrm{BH}$ sources spanning $10^{10}$ orders of magnitude in mass and $10^{6}$ orders of magnitude in luminosity. We found that these luminosities and therefore, the relativistic particle population responsible for them could be due to this magnetic power (see Section 4.3).

Though the specific nature of the particle acceleration mechanism is not a critical point in the present work, a few notes are in order in this regard. Particle acceleration by a first-order Fermi process in fast magnetic reconnection sites has been extensively studied (see, e.g., the reviews in Kowal et al. 2011, 2012; de Gouveia Dal Pino \& Kowal
2015: de Gouveia Dal Pino et al. 2014, and references therein). As remarked before, GL05 were the first to propose that this process might occur within current sheets. In analogy to shock acceleration, GL05 verified that particles trapped between the two converging magnetic fluxes (moving to each other with a velocity $V_{R}$ ), would bounce back and forth undergoing head-on interactions with magnetic fluctuations and their energy after a round trip would increase by $\left\langle\Delta E / E>\sim V_{R} / c\right.$, which implies a first-order Fermi process with an exponential energy growth after several round trips, resulting a power-law particle spectrum. Before that, several authors (e.g., Litvinenko 1996; Shibata \& Tanuma 2001; Zenitani \& Hoshino 2001) addressed the acceleration of energetic particles in reconnection sites but did not describe the process as a first-order Fermi process. The analytical predictions of GL05 were confirmed by Drake et al. (2006) who made 2D numerical PIC simulations of the process in collisionless fluids which thus work only for $2 \mathrm{D}$ converging magnetic islands and probe only kinetic scales (see also, Zenitani \& Hoshino 2008; Zenitani et al. 2009). The equivalence between the two models was discussed in Kowal et al. (2011, 2012) who performed 2D and 3D numerical collisional MHD simulations with test particles. Besides, these authors demonstrated that the process works in 3D fluids (where the 2D magnetic islands are opened into 3D loops, as described in GL05).

These results strengthen the possibility that the overall magnetic reconnection process in the surrounds of a $\mathrm{BH}$, near the jet launching basis, can lead to particle acceleration and allow for the observed Synchrotron ra- 
dio emission in the core regions of these sources and the associated high energy gamma-ray emission as wel[11.

\subsection{Comparison with observations}

Derived as a function of inner radius region parameters, i.e., the mass of the central $\mathrm{BH}\left(m=M / M_{\odot}\right)$, the disk mass accretion rate $\left(\dot{m}=\dot{M} / \dot{M}_{E d d}\right)$, the extension of the coronal loops $\left(l=L / R_{S}\right)$, and the extension of the magnetic reconnection region in the corona $\left(l_{X}=L_{X} / R_{S}\right)$, the calculated fast magnetic reconnection power driven by turbulence was compared with the observed nuclear radio and gamma-ray emission of a much larger sample of compact sources than that used in GPK10, including microquasars, low-luminosity AGNs (LLAGNs, i.e., LINERS and Seyfert galaxies), as well as blazars and GRBs. Our results show that, in general, just a small fraction of this power would be enough to explain the observed radio luminosities of the lowluminosity sources (LLAGNs and microquasars) (see Figure 4). In most of these cases, the corresponding required accretion rate is $\dot{m}<0.05$. Also striking is the fact that the slope dependence of the magnetic power released by turbulent reconnection with the source mass nearly follows the same trend of the observed radio luminositysource mass correlation for these sources (Nagar et al. 2002, 2005; Merloni et al. 2003, see Figure 3), which suggests that this mechanism could provide a natural interpretation for this correlation, as suggested earlier by GPK10, but considering a very small sample of sources and fast reconnection induced by anomalous resistivity only.

The corresponding gamma-ray emission of these sources, which is believed to be produced by a number of leptonic and hadronic processes involving the accelerated electrons and protons, respectively (through synchrotron-self Compton, inverse Compton, protonproton inelastic collisions, and proton-photon collisions (Romero et al. 2003; Khiali et al. 2015), can in principle be also associated with the same emission zone that produces the radio synchrotron emission in the core of these sources. For this reason, we have investigated whether the power released by magnetic reconnection could also be connected with the gamma-ray emission of these sources. We see that this could be the case for microquasars and LLAGNs. The observed gamma-ray luminosity of these sources is nearly correlated with both the radio luminosity and the calculated magnetic reconnection power (Figure 5), being smaller than the latter. This suggests that the accelerated particles by the magnetic reconnection mechanism here discussed can produce the radio emission and be also responsible for the processing of the high energy emission in the core region. Even the radio galaxy IC 310 which has been argued to emit like a blazar (e.g., Aleksić et al. 2014a), follows this trend. We note however that, because in most cases the observed gamma-ray luminosity is larger than the radio luminosity, the former lies, in general, in the upper parts of the magnetic reconnection diagram, therefore corre-

\footnotetext{
11 Of course, as stressed before, we cannot exclude the possibility that fast magnetic reconnection may also lead to the production of plasmoids that can propitiate the formation of a shock right outside of the reconnection region allowing for particle acceleration in this shock (see also Khiali et al. 2015).
}

sponding to accretion rates which are larger than those predicted when examining only the radio emission of the sources. This suggests that in most cases, in order to produce magnetic power enough to accelerate particles able to produce both the radio and the VHE emission, the accretion rates must be in general $\dot{m} \geq 0.05$.

Moreover, the correlations found above may also shed some light in the interpretation of the so-called "fundamental plane" obtained empirically, which correlates the radio and X-ray emission of microquasars and lowluminosity AGNs with the BH mass of the sources (see Merloni et al.|2003; Falcke et al.|2004; Wang et al. 2006; Körding et al. 2006; Li et al. 2008; Yuan et al. 2009; Gültekin et al. 2009; Plotkin et al. 2012; Huang et al. 2014). Although we here did not deal with the X-ray emission which is directly related with the accretion disk processes, but focussed on the radio and VHE emissions related to the disk corona, our model suggests a simple physical interpretation for the existence of these empirical correlations as linked to magnetic reconnection activity in the core of these sources. The fact that fast magnetic reconnection and the associated radio flare is a transient and violent phenomenon that leads to the partial destruction of the equilibrium configuration in the inner accretion disk/corona region in the surrounds of the $\mathrm{BH}$, suggests that this process could be related to the transition from the low/hard to the high/soft steep-power-law (SPL) X-ray states often detected in microquasars (Remillard \& McClintock 2006; Zhang et al. 2014), as argued in GPK10. However, a detailed analysis of this transition and the accretion disk-coronal state that follows an event of fast reconnection, as well as the reproduction of the whole spectral energy distribution of the sources is out of the scope of this work.

Our results change considerably in the case of blazars. Although after Doppler beaming correction, in most cases their radio emission lies within the upper part of the magnetic reconnection power $\dot{W}_{B}$ diagram, which corresponds to accretion rates much larger than $\dot{m}=0.05$, this emission, in general, does not seem to follow the same trend as that of the LLAGNs or of the magnetic reconnection power itself (Figure 6). In the case of their gamma-ray luminosity, even with the Doppler beaming correction, most of this power lies well above the $\dot{W}_{B}$ diagram (Figure 7). Comparing this with the gamma-ray emission of a sample of GRBs, we see that the line that links both the blazars and the GRBs gamma-ray emissions in the diagram is anti-correlated with the gray zone corresponding to the fast magnetic reconnection power in the core. This suggests that the emission in these sources cannot be attributed to this acceleration process. In the case of blazars, for which the jet axis points to the line of sight, this result is consistent with the standard scenario for these sources where the emission is attributed to relativistic particle acceleration along the jet which has relativistic bulk velocities. A similar scenario is applicable to GRBs. Their prompt gamma-ray emission and the afterglow radio emission are attributed to the reverse internal shock and the forward external shock, respectively, of a super relativistically beamed jet after it breaks out from the stellar progenitor envelope (e.g., Woosley 1993; Paczyński 1998; MacFadven \& Wooslev 1999). In other words, in both classes of sources, the observed emission 
seems to be produced at the jet at distances larger than a few $20 R_{S}$ from the core of the sources and probably cannot be explained by the magnetic reconnection scenario here described. Any deep core emission in these sources is probably screened by opacity and by the jet pointing towards our line of sight. This result is consistent with the predictions of GPK10, and also with Nemmen et al. (2012) whose observed correlation between GRBs and blazars suggests that the gamma-ray and radio emission from such sources is originated further out at the associated relativistic jet.

We note that in recent work Zhang \& Yan (2011) invoked the LV99 fast reconnection model and the GL05 first-order Fermi acceleration mechanism to explain the emission features in some GRBs. Specifically, they suggested that the GRB prompt emission would occur in a Poynting-flux dominated regime through the collision of multiple injected shells into the jet flow. These would distort the magnetic field lines and induce fast reconnection which in turn would induce turbulence further distorting the magnetic field lines, easing additional magnetic reconnection and resulting in a runway release of the stored magnetic field energy and particle acceleration. This mechanism is somewhat similar to what we have suggested here in the sense that the onset of instabilities and the continuous reconnection during the building of the corona itself may trigger turbulence which in turn speeds up the overall process. However, distinctly from Zhang \& Yan (2011) model which is a mechanism occurring in the jet beam, our model occurs within the nuclear region of the system. Other works have also investigated the effects of fast reconnection along the jet (e.g., Giannios 2010; Uzdenskv \& McKinney 2011; de Gouveia Dal Pino \& Kowal 2015). In particular, de Gouveia Dal Pino \& Kowal (2015) have injected test particles in an MHD simulation of a relativistic jet and found evidence of efficient particle acceleration by reconnection in this system.

We should stress that the results of the fast magnetic reconnection model investigated here are relatively insensitive to the physical parameters inside the accretion disk, except for the accretion rate which was allowed to vary between $0.0005 \leq \dot{m} \leq 1$. The source mass is the more critical parameter in our model as it varies over $10^{10}$ orders of magnitude. This trend seems to be confirmed when we repeat the calculations here presented but adopting a magnetically dominated advective flow (MDAF or MAD, see Meier 2012; Sikora \& Begelman 2013) to describe the disk accretion/corona in the inner region of the source, rather than the standard ShakuraSunyaev disk. In fact, with this new approach we find that the results and the slope of the diagrams of Figures 4 to 7 do not change substantially (see Singh et al. 2015).

As remarked, other contemporary works have also explored magnetic processes in the surrounds of $\mathrm{BH}$ systems and other compact sources to explain their emission (see, e.g., Soker 2010; Cemeliić et al. 2013; Uzdensky \& Spitkovskv 2014; Huang et al. 2014). In particular Huang et al. (2014) investigated magnetic reconnection in the surrounds of $\mathrm{BH}$ binary systems (microquasars) employing a radiatively inefficient advectiondominated accretion flow (RIAF) to describe the accretion disk combined with a jet model. They find that this could explain the observed correlation between the radio and X-ray emission for the high soft state. Furthermore, they argue that the sources that deviate from this correlation (the outliers) could be explained by an appropriate combination of these two mechanisms, a result that is consistent with the present analysis and the arguing of GPK10.

\subsection{Summary and Final remarks}

In summary, the results of the present work indicate that in the case of microquasars and low luminosity AGNs (LLAGNs), the power released by fast magnetic reconnection driven by turbulence in the surrounds of the $\mathrm{BH}$ is able to explain the observed core radio and gammaray emission of these sources, therefore indicating that the surrounds of the BHs (as sketched in Figure 1) can be the acceleration region in these cases. Also, according to our results, fast reconnection induced by anomalous resistivity is clearly less efficient to provide the appropriate magnetic power for most of the sources of the sample, therefore, fast reconnection induced by turbulence (as described in LV99) is clearly more appropriate and besides, it results nearly the same trend (slope) of the observed luminosity distributions for these sources (see Figures 3to 5). On the other hand, in the case of blazars (and GRBs), our results show that the magnetic power released by fast reconnection (either driven by turbulence or anomalous resistivity) in the surrounds of the central source is clearly not sufficient to explain both the observed radio and gamma-ray radiation for most of these sources (Figures 6] and [7). This is probably due to the fact that these sources have their jets pointing to our line of sight and therefore, the core emission is screened by the jet. So that what is effectively observed is emission coming from further out - from the jet, as generally expected for these sources.

The results above connecting both the radio and gamma-ray emission from low-luminosity compact sources to magnetically dominated reconnection processes are very promising as they suggest a unifying single process of relativistic particle acceleration in the core region which may naturally help with the interpretation of the observed correlations of LLAGNs and microquasars, as remarked, and also with clues for existing unification AGN theories, providing important predictions for the coming new generation of VHE observatories with much larger sensitivity and energy range to perform emission and variability studies, such as the Cherenkov Telescope Array (CTA; see Actis et al. 2011; Acharva et al. 2013; Sol et al. 2013). Also, multi-frequency observation (as, e.g., Hovatta et al. 2014) will be crucial to better constrain the location of the gamma-ray emission and the acceleration mechanisms.

Finally, we should note that in this work we have focussed on the total power released by magnetic reconnection in the core region of the sources, without examining the radiation mechanisms by which this energy can be transformed into radio or gamma-ray emission. In a companion work we have explored the acceleration mechanism above operating in the core region of the microquasars Cyg X-1 and Cyg X-3 (see Figures 4, 5] and 7) and have reproduced their entire observed non-thermal spectral energy distribution (SED), from the radio to the gamma-ray flux profile (see Khiali et al. 2015). 
This work has been partially supported by grants from the Brazilian agencies FAPESP (2013/09065-8, 2013/10559-5), CNPq (142220/2013-2 and 306598/20094) and CAPES. This paper has also benefited from very fruitful discussions with Alex Lazarian, Zulema Abraham, Rodrigo Nemmen, Tsvi Piran, and James Stone. The authors also acknowledge the useful comments from an anonymous referee.

\section{REFERENCES}

Abdo, A.A., et al. 2009, Science, 326, 1512

Abdo, A. A., Ackermann, M., Ajello, M., et al. 2009, ApJ, 707, 55 Abdo, A. A., Ackermann, M., Ajello, M., et al. 2010, ApJ, 719, 1433

Abramowski, A., et al. 2012, ApJ, 746, 151

Ackermann, M., et al. 2012, ApJ, 747, 104

Acharya, B. S., Actis, M., Aghajani, T., et al. 2013, Astroparticle Physics, 43, 3

Actis, M., Agnetta, G., Aharonian, F., et al. 2011, Experimental Astronomy, 32, 193

Aharonian,F., et al. 2003, A\&A, 403, L1

Aharonian,F., et al. 2009, ApJ Letters, 695, L40

Albert, J., et al. 2007, ApJ Letters, 665, 51

Aleksić, J., et al. 2010a, ApJ, 723, L207

Aleksić, J., et al. 2010b, ApJ, 721, 843

Aleksić, J., et al. 2012, A\&A, Letters 539, 2

Aleksić, J., Antonelli, L. A., Antoranz, P., et al. 2014a, A\&A, 563, A91

Aleksić, J., Ansoldi, S., Antonelli, L. A., et al. 2014b, A\&A, 564, A5

Arons, J. 1993, ApJ, 408, 160

Balbus, S. A., \& Hawley J. F., 1998, RvMP, 70, 1

Bhattacharjee, A., \& Hameiri, E. 1986, Physical Review Letters, 57, 206

Bhattacharjee, A., Huang, Y.-M., Yang, H., \& Rogers, B. 2009, Physics of Plasmas, 16, 112102

Biskamp, D., Schwarz, E., \& Drake, J. F. 1997, Physics of Plasmas, 4, 1002

Blandford, R. D., \& Payne, D. G., 1982, MNRAS, 199, 883

Blandford, R. D., \& Rees, M. J., 1974, MNRAS, 169, 395

Blandford, R. D., \& Znajek, R. L., 1977, MNRAS, 179, 433

Bodaghee, A., Tomsick, J. A., Pottschmidt, K., et al. 2013, ApJ, 775,98

Cemeljić, M., Shang, H., \& Chiang, T.-Y. 2013, ApJ, 768, 5

Chatterjee, P., Hernquist, L., \& Narayan, R. 2000, ApJ, 534,373

Cho, J., \& Lazarian, A. 2014, ApJ, 780, 30

de Gouveia Dal Pino, E.M., \& Lazarian, A. 2000,ApJ, 536, 31

de Gouveia Dal Pino, E.M., \& Lazarian, A. 2005, A\&A, 441, 845

de Gouveia Dal Pino, E.M., Piovezan, P.P., \& Kadowaki, L.H.S. 2010a, A\&A, 518, A5

de Gouveia Dal Pino, E.M., Kowal, G., Kadowaki, L.H.S.,

Piovezan, P., \& Lazarian, A. 2010b, International Journal of Modern Physics D, 19, 729

de Gouveia Dal Pino, E.M., \& Kowal, G. 2015, in Magnetic Fields in Diffuse Media, A. Lazarian. E. de Gouveia Dal Pino, C. Melioli (eds.), Astrophysics and Space Science Library, 407, 373, arXiv1302.4374D

de Gouveia Dal Pino, E.M., Kowal, G., \& Lazarian, A. 2014, 8th International Conference of Numerical Modeling of Space Plasma Flows (ASTRONUM 2013), ASP Conference Series, Vol. 488 , p. 8

del Valle, M. V. et al., 2014, in prep.

Dexter, J., McKinney, J. C., Markoff, S., \& Tchekhovskoy, A. 2014, MNRAS, 440, 2185

Dhawan, V., Mirabel, I. F., \& Rodríguez, L. F., 2000, ApJ, 543, 3735

Diamond, P. H., \& Malkov, M. 2003, Physics of Plasmas, 10, 2322

Drake, J. F., Swisdak, M., Che, H., \& Shay, M. A. 2006, Nature, 443,553

Drake, J. F., Opher, M., Swisdak, M., \& Chamoun, J. N. 2010, ApJ, 709, 963

Eyink, G. L., Lazarian, A., \& Vishniac, E. T. 2011, ApJ, 743, 51

Eyink, G., Vishniac, E., Lalescu, C., et al. 2013, Nature, 497, 466

Falcke, H., Krding, E., \& Markoff, S., 2004, A\&A, 414, 895
Fender, R. P., Belloni, T. M., \& Gallo, E., 2004, MNRAS, 355, 1105

Fender, R., \& Belloni, T. 2012, Science, 337, 540

Frank, J., King, A., \& Raine, D. J. 2002, Accretion Power in Astrophysics, by Juhan Frank and Andrew King and Derek Raine, pp. 398. ISBN 0521620538. Cambridge, UK: Cambridge University Press, February 2002.

Gebhardt, K., Bender, R., Bower, G., et al. 2000, ApJ, 539, L13

Giannios, D. 2010, MNRAS, 408, L46

Grier, C. J., Peterson, B. M., Pogge, R. W., et al. 2012, ApJ, 755, 60

Gültekin, K., Cackett, E. M., Miller, J. M., et al. 2009, ApJ, 706, 404

Guo, Z. B., Diamond, P. H., \& Wang, X. G. 2012, ApJ, 757, 173

Hameiri, E., \& Bhattacharjee, A. 1987, Physics of Fluids, 30,

Hannikainen, D., Wu, K., Campbell-Wilson, D., et al., 2001,

Exploring the Gamma-Ray Universe, 459, 291

Ho, L. C., Filippenko, A. V., \& Sargent, W. L. W. 1997, ApJS, 112,315

Hovatta, T., Pavlidou, V., King, O. G., et al. 2014, MNRAS, 439, 690

Huang, C.-Y., Wu, Q., \& Wang, D.-X. 2014, MNRAS, 440, 965

Igumenshchev, I. V. 2009, ApJ, 702, L72

Israel, F. P. 1998, A\&A Rev., 8, 237

Jacobson, A. R., \& Moses, R. W. 1984, Phys. Rev. A, 29, 3335

Kadler, M., Eisenacher, D., Ros, E., et al. 2012, A\&A, 538, L1

Kharb, P., Lister, M. L., \& Cooper, N. J. 2010, ApJ, 710, 764

Khiali, B., de Gouveia Dal Pino, E. M., \& del Valle, M. V. 2015, MNRAS(accepted), arXiv:1406.5664

King, A. R., Pringle, J. E., West, R. G., \& Livio, M., 2004 MNRAS, 348, 111

King, A. R., Pringle, J. E., \& Livio, M., 2007, MNRAS, 376, 1740

Kowal, G., Lazarian, A., Vishniac, E. T., Otmianowska-Mazur, K., 2009, ApJ, 700, 63

Kowal, G., de Gouveia Dal Pino, E.M., \& Lazarian, A. 2011, ApJ, 735, 102

Kowal, G., de Gouveia Dal Pino, E.M., \& Lazarian, A. 2012,

Physical Review Letters, 108, 241102

Körding, E., Falcke, H., \& Corbel, S. 2006, A\&A, 456, 439

Krolik, J. H., \& Piran, T. 2011, ApJ, 743, 134

Krolik, J. H., \& Piran, T. 2012, ApJ, 749, 92

Kulsrud, R. M. 1983, Basic Plasma Physics: Selected Chapters,

Handbook of Plasma Physics, Volume 1, 1

Lazarian, A., \& Vishniac, E., 1999, ApJ, 517, 700

Lazarian, A. 2011, arXiv:1111.0694

Lazarian, A., Vlahos, L., Kowal, G., Yan, H.; Beresnyak, A.; de Gouveia Dal Pino, E. M. 2012, Space Sci. Rev., 173, 557

Lazarian, A., Eyink, G. L., Vishniac, E. T., \& Kowal, G. 2015, in Magnetic Fields in Diffuse Media (A. Lazarian, E. M. de Gouveia Dal Pino, C. Melioli eds.), Astrophysics and Space Science Library, 407, 311

Li, Z.-Y., Wu, X.-B., \& Wang, R. 2008, ApJ, 688, 826

Lin, R. P., \& Hudson, H. S. 1971, Sol. Phys., 17, 412

Liu, B.F., Mineshige, S., \& Shibata, K. 2002, ApJ, 572, 173

Liu, B.F., Mineshige, S., \& Ohsuga , K. 2003, ApJ, 587, 571

Litvinenko, Y. E. 1996, ApJ, 462, 997

Livio, M., Pringle, J. E.,\& King, A. R., 2003, ApJ, 593, 184

Loureiro, N. F., Uzdensky, D. A., Schekochihin, A. A., Cowley, S. C., \& Yousef, T. A. 2009, MNRAS, 399, L146

Lynden-Bell, D., 1969, Nature, 223, 690

Lyne, A. G., \& Graham-Smith, F. 1990, Pulsar astronomy", Cambridge University Press

Lyutikov, M., \& Lazarian, A. 2013, Space Sci. Rev., 178, 459

MacDonald, D. A., Thorne, K. S., Price, R. H., \& Zhang, X. H., 1986, in Black Holes: The Membrane Paradigm, 120

MacFadyen, A. I., \& Woosley, S. E. 1999, ApJ, 524, 262

Malyshev, D., Zdziarski, A. A., \& Chernyakova, M. 2013, MNRAS, 434, 2380

Matthaeus, W. H., \& Lamkin, S. L. 1985, Physics of Fluids, 28, 303

Matthaeus, W. H., \& Lamkin, S. L. 1986, Physics of Fluids, 29, 2513

McKinney, J. C., \& Blandford, R. D., 2009, MNRAS, 394, 126

McKinney, J. C., Tchekhovskoy, A., \& Blandford, R. D. 2012,

MNRAS, 423,3083 
Meier, D. L. 2012, Black Hole Astrophysics: The Engine Paradigm, by David L. Meier. ISBN: 978-3-642-01935-7. Springer, Verlag Berlin Heidelberg, 2012,

Menou, K., Perna, R., \& Hernquist, L. 2001, ApJ, 554, L63

Merloni, A., Heinz, S., \& di Matteo, T. 2003, MNRAS, 345,1057

Merritt, D., \& Ferrarese, L. 2001, MNRAS, 320, L30

Middleton, M., Done, C., \& Schurch, N. 2008, MNRAS, 383, 1501

Mirabel, I. F., \& Rodríguez, L. F., 1994, Nature, 371, 46

Mirabel, I. F., \& Rodríguez, L. F. 1998, Nature, 392, 673

Nagar, N. M., Falcke, H., Wilson, A. S., \& Ulvestad, J. S. 2002, A\&A, 392, 53

Nagar, N.M., Falcke, H., \& Wilson, A.S. 2005, A\&A, 435, 521

Nemmen, R.S., et al. 2012, Science, 338, 1445

Neronov, A., \& Aharonian, F. A. 2007, ApJ, 671, 85

Paczyński, B. 1998, Gamma-Ray Bursts, 4th Hunstville Symposium, 428, 783

Papadopoulos, K. 1977, Reviews of Geophysics and Space Physics, 15, 113

Parker, E. N. 1957, J. Geophys. Res., 62, 509

Parker, E. N. 1979, Oxford, Clarendon Press; New York, Oxford University Press, 1979, 858 p.,

Piano, G., Tavani, M., Vittorini, V., et al. 2012, A\&A, 545, A110

Plotkin, R. M., Markoff, S., Kelly, B. C., Körding, E., \& Anderson, S. F. 2012, MNRAS, 419, 267

Remillard, R. A., \& McClintock, J. E., 2006, ARA\&A, 44, 49

Reynoso, M. M., Medina, M. C., \& Romero, G. E. 2011, A\&A, 531, A30

Richstone, D., Ajhar, E. A., Bender, R., et al. 1998, Nature, 395, A14

Rieger, F. M., \& Aharonian, F. A. 2008, A\&A, 479, L5

Romanova, M. M., Ustyugova, G. V., Koldoba, A. V., \& Lovelace, R. V. E. 2002, ApJ, 578, 420

Romanova, M. M., Ustyugova, G. V., Koldoba, A. V., \& Lovelace, R. V. E. 2011, MNRAS, 416, 416

Romero, G. E., Torres, D. F., Kaufman Bernadó, M. M., \& Mirabel, I. F. 2003, A\&A, 410, L1

Romero, G.E., et al. 2007, in Relativistic Astrophysics Legacy and Cosmology Einsteins ESO Astrophysics Symposia, 480

Shakura, N.I., \& Sunyaev, R.A. 1973, A\&A 24, 337

Shay, M. A., Drake, J. F., Denton, R. E., \& Biskamp, D. 1998, J. Geophys. Res., 103, 9165

Shay, M. A., Drake, J. F., Swisdak, M., \& Rogers, B. N. 2004, Physics of Plasmas, 11, 2199

Shibata, K., \& Tanuma, S. 2001, Earth, Planets, and Space, 53, 473

Speiser, T. W. 1970, Planet. Space Sci., 18, 613

Spitzer, L. 1962, Physics of Fully Ionized Gases, New York: Interscience (2nd edition), 1962,

Sikora, M., \& Begelman, M. C. 2013, ApJ, 764, L24

Singh, C. B., de Gouveia Dal Pino, E. M., \& Kadowaki, L. H. S. 2015, ApJ, 799, LL20

Sol, H., Zech, A., Boisson, C., et al. 2013, Astroparticle Physics, 43,215
Soker, N. 2010, ApJ, 721, L189

Somov, B. V. 2012,Plasma Astrophysics, Part I: Fundamentals and Practice, Astrophysics and Space Science Library, 391,

Stone, J. M., Hawley, J. F., Gammie, C. F., \& Balbus, S. A. 1996, ApJ, 463, 656

Strauss, H. R. 1986, Physical Review Letters, 57, 2231

Strauss, H. R. 1988, ApJ, 326, 412

Tagger, M., Varnière, P., Rodriguez, J., \& Pellat, R. 2004, ApJ, 607,410

Takasaki, H., Asai A., Kiyohara, J., et al. 2004, ApJ, 613, 592

Tavani,M., et al. 2011, Science, 331, 736

Tavani,M. , et al. 2009, Nature, 462, 620

Tavecchio, F., \& Ghisellini, G. 2008, MNRAS, 385, L98

Tavecchio, F., \& Ghisellini, G. 2008, MNRAS, 386, 945

Tchekhovskoy, A., Narayan, R., \& McKinney, J. C. 2011, MNRAS, 418, L79

Tchekhovskoy, A., Metzger, B. D., Giannios, D., \& Kelley, L. Z 2014, MNRAS, 437, 2744

Tremaine, S., Gebhardt, K., Bender, R., et al. 2002, ApJ, 574, 740

Uzdensky, D. A. 2011, Space Sci. Rev., 160, 45

Uzdensky, D. A., Goodman, J., 2008, ApJ, 682, 608

Uzdensky, D. A., \& McKinney, J. C. 2011, Physics of Plasmas, 18,042105

Uzdensky, D. A., \& Spitkovsky, A. 2014, ApJ, 780, 3

Vovk, I., \& Neronov, A. 2013, ApJ, 767, 103

Xu, S., \& Yan, H. 2013, ApJ, 779, 140

Waelbroeck, F. L. 1989, Physics of Fluids B, 1, 2372

Wang, D. X., Xiao, K., \& Lei, W. H., 2002, MNRAS, 335, 65

Wang, R., Wu, X.-B., \& Kong, M.-Z. 2006, ApJ, 645, 890

Winter, L. M., Mushotzky, R. F., Reynolds, C. S., \& Tueller, J. 2009, ApJ, 690, 1322

Woosley, S. E. 1993, ApJ, 405, 273

Yamada, M., Kulsrud, R., \& Ji, H. 2010, Reviews of Modern Physics, 82, 603

Yamada, M. et al. Conversion of magnetic energy in the magnetic reconnection layer of a laboratory plasma. Nat. Commun. 5:4774 doi: 10.1038/ncomms5774 (2014)

Yokoi, N., \& Hoshino, M. 2011, Physics of Plasmas, 18, 111208

Yuan, F., Yu, Z., \& Ho, L. C. 2009, ApJ, 703, 1034

Zamaninasab, M., Clausen-Brown, E., Savolainen, T., \& Tchekhovskoy, A. 2014, Nature, 510, 126

Zanni, C., \& Ferreira, J. 2009, A\&A, 508, 1117

Zanni, C., \& Ferreira, J. 2013, A\&A, 550, A99

Zenitani, S., \& Hoshino, M. 2001, ApJ, 562, L63

Zenitani, S., \& Hoshino, M. 2008, ApJ, 677, 530

Zenitani, S., Hesse, M., \& Klimas, A. 2009, ApJ, 696, 1385

Zhang, B., \& Yan, H. 2011, ApJ, 726, 90

Zhang, J., Xu, B., \& Lu, J. 2014, ApJ, 788, 143

\section{APPENDIX}

\section{A. GAS AND RADIATION PRESSURE REGIMES}

Figure 8 presents the ratio between the radiation and the gas pressure in the accretion disk versus the source mass for different values of the accretion rate, as obtained from equations (16) and (17).

\section{B. SUPPLEMENTARY TABLES}

Table 1 Radio and gamma-ray emission (when available) of LLAGNs and microquasars (or galactic black hole binaries).

\begin{tabular}{|c|c|c|c|c|c|c|c|c|}
\hline $\begin{array}{l}\text { Sources } \\
(1)\end{array}$ & $\begin{array}{c}\text { Type } \\
(2)\end{array}$ & $\begin{array}{c}\log _{10}\left(L_{R}[e r g / s]\right) \\
(3)\end{array}$ & $\begin{array}{c}\text { Notes } \\
(4)\end{array}$ & $\begin{array}{cc}\text { Ref. } & \log _{10}\left(L_{\gamma}[e r g / s]\right) \\
(5) & (6)\end{array}$ & $\begin{array}{l}\text { Notes } \\
(7)\end{array}$ & $\begin{array}{c}\text { Ref. } \\
(8)\end{array}$ & $\begin{array}{c}\log _{10}\left(M / M_{\odot}\right) \\
(9)\end{array}$ & $\begin{array}{l}\text { Ref. } \\
(10)\end{array}$ \\
\hline IC 239 & $\mathrm{~L}$ & 36.66 & $\mathrm{UL}$ & {$[0]$} & & & 6.67 & {$[3][4]$} \\
\hline IC 356 & $\mathrm{~T}$ & 36.77 & UL & {$[0]$} & & & 7.69 & [3] $[4]$ \\
\hline IC 520 & $\mathrm{~T}$ & 37.60 & UL & {$[0]$} & & & 7.44 & [3] $[4]$ \\
\hline IC 1727 & $\mathrm{~T} / \mathrm{L}$ & 36.04 & $\mathrm{UL}$ & {$[0]$} & & & 7.41 & {$[3][4]$} \\
\hline NGC 185 & $\mathrm{~S}$ & 33.99 & UL & $0]$ & & & 3.89 & [3] $[4]$ \\
\hline NGC 266 & $\mathrm{~L}$ & 38.46 & & {$[0]$} & & & 8.39 & {$[3][4]$} \\
\hline
\end{tabular}




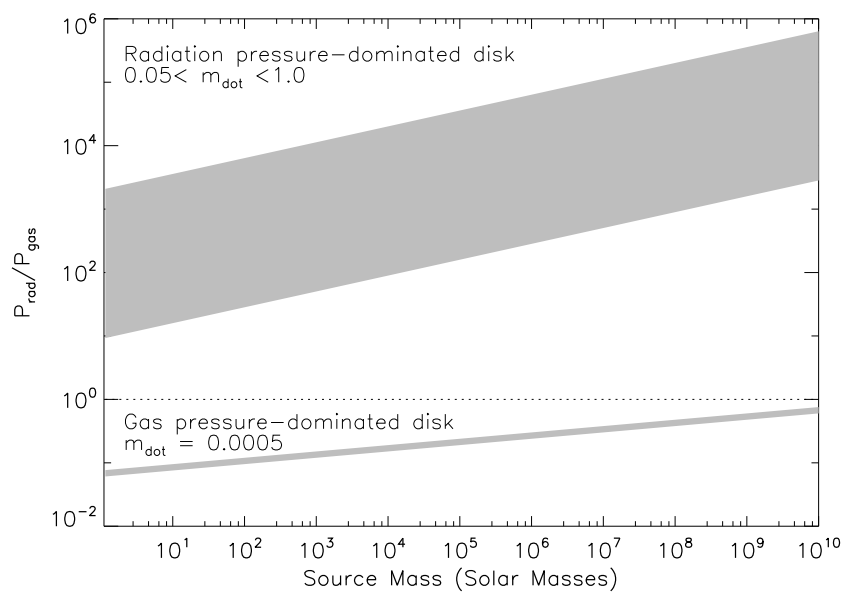

Figure 8. Ratio between the radiation and the gas pressure of the accretion disk $\left(P_{\text {rad }} / P_{\text {gas }}\right)$ as a function of the source mass for different values of $\dot{m}$. The gray regions give the parametric space for $P_{\text {rad }} / P_{\text {gas }}$ for the radiation pressure (top) and the gas pressure dominated (bottom) regimes. The parametric space considered is $0.05 \leq \alpha \leq 0.5, \dot{m} \simeq 5 \times 10^{-4}$ for the gas-pressure dominated regime, and $5 \times 10^{-2}<\dot{m}<1$ for the radiation-pressure dominated regime.

\begin{tabular}{|c|c|c|c|c|c|c|c|c|c|}
\hline $\begin{array}{l}\text { Sources } \\
(1)\end{array}$ & $\begin{array}{c}\text { Type } \\
(2)\end{array}$ & $\begin{array}{c}\log _{10}\left(L_{R}[\mathrm{erg} / \mathrm{s}]\right) \\
(3)\end{array}$ & $\begin{array}{l}\text { Notes } \\
(4)\end{array}$ & $\begin{array}{l}\text { Ref. } \\
(5)\end{array}$ & $\begin{array}{c}\log _{10}\left(L_{\gamma}[\mathrm{erg} / \mathrm{ss}]\right) \\
(6)\end{array}$ & $\begin{array}{l}\text { Notes } \\
(7)\end{array}$ & $\begin{array}{l}\text { Ref. } \\
(8)\end{array}$ & $\begin{array}{c}\log _{10}\left(M / M_{\odot}\right) \\
(9)\end{array}$ & $\begin{array}{l}\text { Ref. } \\
(10)\end{array}$ \\
\hline NGC 315 & $\mathrm{~L}$ & 40.57 & & {$[0]$} & & & & 8.96 & {$[3][4][5]$} \\
\hline NGC 404 & $\mathrm{~L}$ & 35.13 & UL & {$[0]$} & & & & 4.84 & [3][4] \\
\hline NGC 410 & $\mathrm{~L}$ & 37.96 & UL & {$[0]$} & & & & 8.88 & {$[3][4]$} \\
\hline NGC 428 & $\mathrm{~T} / \mathrm{L}$ & 36.56 & UL & {$[0]$} & & & & 4.36 & [3] 4$]$ \\
\hline NGC 474 & $\mathrm{~L}$ & 37.46 & UL & {$[0]$} & & & & 7.66 & [3] [4] \\
\hline NGC 488 & $\mathrm{~T}$ & 37.19 & UL & {$[0]$} & & & & 8.06 & [3] [4] \\
\hline NGC 521 & $\mathrm{~T} / \mathrm{H}$ & 37.91 & UL & {$[0]$} & & & & 8.23 & [3][4] \\
\hline NGC 524 & $\mathrm{~T}$ & 37.45 & & {$[0]$} & & & & 8.48 & [3] [4] \\
\hline NGC 660 & $\mathrm{~T} / \mathrm{H}$ & 36.36 & UL & {$[0]$} & & & & 7.10 & [3] [4] \\
\hline NGC 676 & $\mathrm{~S}$ & 37.01 & UL & {$[0]$} & & & & 7.55 & {$[3][4]$} \\
\hline NGC 718 & $\mathrm{~L}$ & 37.09 & UL & {$[0]$} & & & & 7.15 & {$[3][4]$} \\
\hline NGC 777 & $\mathrm{~S} / \mathrm{L}$ & 38.08 & UL & {$[0]$} & & & & 9.01 & [3] $[4]$ \\
\hline NGC 841 & $\mathrm{~L}$ & 37.98 & UL & {$[0]$} & & & & 7.70 & [3] 4$]$ \\
\hline NGC 1055 & $\mathrm{~T} / \mathrm{L}$ & 36.71 & UL & {$[0]$} & & & & 6.41 & [3] 4$]$ \\
\hline NGC 1058 & $\mathrm{~S}$ & 36.13 & UL & {$[0]$} & & & & 5.47 & [3] [4] \\
\hline NGC 1167 & $\mathrm{~S}$ & 39.54 & & {$[0]$} & & & & 8.16 & [3] [4] \\
\hline NGC 1169 & $\mathrm{~L}$ & 37.49 & UL & {$[0]$} & & & & 7.93 & [3] 4$]$ \\
\hline NGC 1275 (Per A) & $\mathrm{S}$ & 41.42 & & {$[0]$} & 41.63 & & {$[13]$} & 8.62 & [3][4][5] \\
\hline NGC 1275 (Per A) & $\mathrm{S}$ & 41.42 & & $0]$ & 43.22 & & {$[13]$} & 8.62 & [3] $[4][5]$ \\
\hline NGC 1961 & $\mathrm{~L}$ & 37.88 & UL & {$[0]$} & & & & 8.50 & {$[3][4]$} \\
\hline NGC 2273 & $\mathrm{~S}$ & 37.78 & & {$[0]$} & & & & 7.43 & {$[3][4][5]$} \\
\hline NGC 2336 & $\mathrm{~L} / \mathrm{S}$ & 37.49 & UL & {$[0]$} & & & & 7.24 & {$[3][4]$} \\
\hline NGC 2541 & $\mathrm{~T} / \mathrm{H}$ & 36.31 & UL & {$[0]$} & & & & 5.65 & {$[3][4]$} \\
\hline NGC 2655 & $\mathrm{~S}$ & 37.81 & & {$[0]$} & & & & 7.71 & {$[3][4]$} \\
\hline NGC 2681 & L & 36.66 & UL & {$[0]$} & & & & 7.21 & [3] [4] \\
\hline NGC 2683 & $\mathrm{~L} / \mathrm{S}$ & 35.72 & UL & {$[0]$} & & & & 7.21 & [3] [4] \\
\hline NGC 2685 & $\mathrm{~S} / \mathrm{T}$ & 36.63 & UL & $0]$ & & & & 6.82 & [3] 4$]$ \\
\hline NGC 2768 & $\mathrm{~L}$ & 37.91 & & {$[0]$} & & & & 7.98 & {$[3][4]$} \\
\hline NGC 2787 & $\mathrm{~L}$ & 37.33 & & {$[0]$} & & & & 7.97 & {$[3][4][5]$} \\
\hline NGC 2832 & $\mathrm{~L}$ & 38.36 & UL & {$[0]$} & & & & 9.09 & [3][4] \\
\hline NGC 2841 & $\mathrm{~L}$ & 36.46 & & {$[0]$} & & & & 8.31 & [3][4][5] \\
\hline NGC 2859 & $\mathrm{~T}$ & 37.07 & UL & $0]$ & & & & 7.92 & {$[3][4]$} \\
\hline NGC 2911 & $\mathrm{~L}$ & 38.75 & & {$[0]$} & & & & 8.47 & [3] $[4]$ \\
\hline NGC 2985 & $\mathrm{~T}$ & 36.96 & UL & {$[0]$} & & & & 7.84 & {$[3][4]$} \\
\hline NGC 3031 & $\mathrm{~S}$ & 37.59 & & {$[0]$} & & & & 7.73 & [3][4][5] \\
\hline NGC 3079 & $\mathrm{~S}$ & 38.56 & & {$[0]$} & & & & 7.83 & [3] [4] [5] \\
\hline NGC 3147 & $\mathrm{~S}$ & 38.38 & & {$[0]$} & & & & 8.55 & {$[3][4][5]$} \\
\hline NGC 3166 & $\mathrm{~L}$ & 37.12 & UL & {$[0]$} & & & & 7.60 & [3][4] \\
\hline NGC 3169 & $\mathrm{~L}$ & 37.68 & & {$[0]$} & & & & 7.96 & [3][4][5] \\
\hline NGC 3190 & $\mathrm{~L}$ & 37.00 & & $0]$ & & & & 8.01 & {$[3][4]$} \\
\hline NGC 3193 & $\mathrm{~L}$ & 37.17 & UL & {$[0]$} & & & & 8.08 & {$[3][4]$} \\
\hline NGC 3226 & $\mathrm{~L}$ & 37.70 & & {$[0]$} & & & & 8.14 & [3][4][5] \\
\hline NGC 3227 & $\mathrm{~S}$ & 37.43 & & {$[0]$} & 41.30 & UL & {$[8]$} & 7.46 & [3] $[4][5]$ \\
\hline NGC 3245 & $\mathrm{~T}$ & 36.95 & UL & $0]$ & & & & 8.21 & {$[3][4]$} \\
\hline NGC 3254 & $\mathrm{~S}$ & 37.18 & UL & {$[0]$} & & & & 7.34 & {$[3][4]$} \\
\hline NGC 3301 & $\mathrm{~L}$ & 37.17 & UL & {$[0]$} & & & & 7.21 & [3] [4] \\
\hline NGC 3368 & $\mathrm{~L}$ & 36.07 & UL & {$[0]$} & & & & 7.28 & [3][4] \\
\hline NGC 3379 & $\mathrm{~L} / \mathrm{T}$ & 36.07 & UL & {$[0]$} & & & & 8.06 & {$[1][2][3][4]$} \\
\hline
\end{tabular}




\begin{tabular}{|c|c|c|c|c|c|c|c|c|c|}
\hline $\begin{array}{l}\text { Sources } \\
(1)\end{array}$ & $\begin{array}{c}\text { Type } \\
(2)\end{array}$ & $\begin{array}{c}\log _{10}\left(L_{R}[e r g / s]\right) \\
(3)\end{array}$ & $\begin{array}{c}\text { Notes } \\
(4)\end{array}$ & $\begin{array}{c}\text { Ref. } \\
(5)\end{array}$ & $\begin{array}{c}\log _{10}\left(L_{\gamma}[e r g / s]\right) \\
(6)\end{array}$ & $\begin{array}{l}\text { Notes } \\
(7)\end{array}$ & $\begin{array}{c}\text { Ref. } \\
(8)\end{array}$ & $\begin{array}{c}\log _{10}\left(M / M_{\odot}\right) \\
(9)\end{array}$ & $\begin{array}{l}\text { Ref. } \\
(10)\end{array}$ \\
\hline NGC 3414 & $\mathrm{~L}$ & 37.41 & & [0] & & & & 8.46 & {$[3][4]$} \\
\hline NGC 3433 & $\mathrm{~L} / \mathrm{T}$ & 37.63 & UL & {$[0]$} & & & & 6.41 & {$[3][4]$} \\
\hline NGC 3486 & $\mathrm{~S}$ & 36.00 & UL & $0]$ & & & & 5.85 & {$[3][4]$} \\
\hline NGC 3489 & $\mathrm{~T} / \mathrm{S}$ & 35.87 & UL & {$[0]$} & & & & 6.91 & {$[3][4]$} \\
\hline NGC 3507 & $\mathrm{~L}$ & 37.03 & UL & {$[0]$} & & & & 6.54 & {$[3][4]$} \\
\hline NGC 3516 & $\mathrm{~S}$ & 37.55 & & {$[0]$} & 41.70 & UL & {$[8]$} & 7.58 & {$[3][4][5]$} \\
\hline NGC 3607 & $\mathrm{~L}$ & 37.06 & & $0]$ & & & & 8.34 & {$[3][4]$} \\
\hline NGC 3608 & $\mathrm{~L} / \mathrm{S}$ & 37.17 & UL & {$[0]$} & & & & 8.08 & {$[2][3][4]$} \\
\hline NGC 3623 & $\mathrm{~L}$ & 35.94 & $\mathrm{UL}$ & $0]$ & & & & 7.54 & {$[3][4]$} \\
\hline NGC 3626 & $\mathrm{~L}$ & 37.27 & $\mathrm{UL}$ & {$[0]$} & & & & 7.48 & {$[3][4]$} \\
\hline NGC 3627 & $\mathrm{~T} / \mathrm{S}$ & 35.94 & & {$[0]$} & & & & 7.26 & {$[3][4][5]$} \\
\hline NGC 3628 & $\mathrm{~T}$ & 36.21 & & {$[0]$} & & & & 6.45 & {$[3][4]$} \\
\hline NGC 3642 & $\mathrm{~L}$ & 37.24 & UL & {$[0]$} & & & & 6.95 & [3][4] \\
\hline NGC 3675 & $\mathrm{~T}$ & 36.47 & $\mathrm{UL}$ & {$[0]$} & & & & 7.00 & {$[3][4][5]$} \\
\hline NGC 3681 & $\mathrm{~T}$ & 37.03 & $\mathrm{UL}$ & {$[0]$} & & & & 6.67 & {$[3][4]$} \\
\hline NGC 3692 & $\mathrm{~T}$ & 37.27 & UL & {$[0]$} & & & & 7.07 & [3] $[4]$ \\
\hline NGC 3705 & $\mathrm{~T}$ & 36.79 & $\mathrm{UL}$ & {$[0]$} & & & & 7.05 & [3] $[4]$ \\
\hline NGC 3718 & $\mathrm{~L}$ & 37.74 & & $0]$ & & & & 7.71 & {$[3][4]$} \\
\hline NGC 3735 & $\mathrm{~S}$ & 37.66 & UL & $0]$ & & & & 7.46 & {$[3][4]$} \\
\hline NGC 3780 & $\mathrm{~L}$ & 37.44 & & {$[0]$} & & & & 6.63 & [3] 4$]$ \\
\hline NGC 3898 & $\mathrm{~T}$ & 36.94 & UL & {$[0]$} & & & & 8.14 & [3] [4] \\
\hline NGC 3900 & $\mathrm{~L}$ & 37.37 & $\mathrm{UL}$ & {$[0]$} & & & & 7.45 & {$[3][4]$} \\
\hline NGC 3917 & $\mathrm{~T}$ & 36.81 & $\mathrm{UL}$ & {$[0]$} & & & & 5.04 & {$[3][4]$} \\
\hline NGC 3941 & $\mathrm{~S}$ & 36.85 & $\mathrm{UL}$ & $0]$ & & & & 7.34 & {$[3][4]$} \\
\hline NGC 3945 & $\mathrm{~L}$ & 37.22 & & {$[0]$} & & & & 7.97 & [3] [4] \\
\hline NGC 3953 & $\mathrm{~T}$ & 36.82 & UL & $0]$ & & & & 7.30 & {$[3][4]$} \\
\hline NGC 3976 & $\mathrm{~S}$ & 37.59 & $\mathrm{UL}$ & {$[0]$} & & & & 8.03 & {$[3][4]$} \\
\hline NGC 3982 & $\mathrm{~S}$ & 36.72 & $\mathrm{UL}$ & $0]$ & & & & 6.16 & {$[3][4]$} \\
\hline NGC 3992 & $\mathrm{~T}$ & 36.82 & UL & {$[0]$} & & & & 7.62 & {$[3][4]$} \\
\hline NGC 3998 & $\mathrm{~L}$ & 38.68 & & {$[0]$} & & & & 8.72 & {$[3][4][5]$} \\
\hline NGC 4013 & $\mathrm{~T}$ & 36.72 & UL & {$[0]$} & & & & 6.54 & {$[3][4]$} \\
\hline NGC 4036 & $\mathrm{~L}$ & 37.22 & $\mathrm{UL}$ & $0]$ & & & & 8.15 & [3][4] \\
\hline NGC 4051 & $\mathrm{~S}$ & 36.72 & UL & {$[0]$} & 40.60 & UL & [8] & 6.63 & {$[3][4][5]$} \\
\hline NGC 4111 & $\mathrm{~L}$ & 36.82 & $\mathrm{UL}$ & {$[0]$} & & & & 7.57 & {$[3][4]$} \\
\hline NGC 4125 & $\mathrm{~T}$ & 37.03 & $\mathrm{UL}$ & {$[0]$} & & & & 8.48 & {$[3][4]$} \\
\hline NGC 4138 & $\mathrm{~S}$ & 36.89 & & {$[0]$} & 40.60 & UL & [8] & 7.28 & [3][4] \\
\hline NGC 4143 & $\mathrm{~L}$ & 37.24 & & {$[0]$} & & & & 8.21 & {$[3][4][5]$} \\
\hline NGC 4150 & $\mathrm{~T}$ & 36.23 & UL & {$[0]$} & & & & 6.50 & {$[3][4]$} \\
\hline NGC 4151 & $\mathrm{~S}$ & 37.97 & & $0]$ & 40.30 & UL & {$[8]$} & 7.00 & {$[3][4][5]$} \\
\hline NGC 4168 & $\mathrm{~S}$ & 37.19 & & $0]$ & & & & 7.96 & {$[3][4]$} \\
\hline NGC 4169 & $\mathrm{~S}$ & 37.74 & & {$[0]$} & & & & 7.97 & [3] $[4]$ \\
\hline NGC 4192 & $\mathrm{~T}$ & 36.82 & UL & {$[0]$} & & & & 7.37 & [3][4] \\
\hline NGC 4203 & $\mathrm{~L}$ & 37.21 & & {$[0]$} & & & & 7.80 & {$[3][4][5]$} \\
\hline NGC 4216 & $\mathrm{~T}$ & 36.79 & & $0]$ & & & & 8.14 & {$[3][4]$} \\
\hline NGC 4220 & $\mathrm{~T}$ & 36.88 & UL & {$[0]$} & & & & 7.02 & {$[3][4]$} \\
\hline NGC 4258 & $\mathrm{~S}$ & 36.34 & & {$[0]$} & & & & 7.53 & {$[1][2][3][4][5]$} \\
\hline NGC 4261 & $\mathrm{~L}$ & 39.83 & & {$[0]$} & & & & 8.80 & [1][2][3][4][5] \\
\hline NGC 4278 & $\mathrm{~L}$ & 38.18 & & {$[0]$} & & & & 8.86 & [3][4][5] \\
\hline NGC 4281 & $\mathrm{~T}$ & 37.35 & UL & {$[0]$} & & & & 8.61 & {$[3][4]$} \\
\hline NGC 4293 & $\mathrm{~L}$ & 36.56 & & {$[0]$} & & & & 7.13 & {$[3][4]$} \\
\hline NGC 4314 & $\mathrm{~L}$ & 36.23 & UL & {$[0]$} & & & & 6.98 & {$[3][4]$} \\
\hline NGC 4321 & $\mathrm{~T}$ & 36.66 & $\mathrm{UL}$ & {$[0]$} & & & & 6.70 & {$[3][4][5]$} \\
\hline NGC 4324 & $\mathrm{~T}$ & 37.35 & $\mathrm{UL}$ & $0]$ & & & & 6.75 & {$[3][4]$} \\
\hline NGC 4346 & $\mathrm{~L}$ & 36.72 & UL & $0]$ & & & & 7.45 & {$[3][4]$} \\
\hline NGC 4350 & $\mathrm{~T}$ & 36.66 & $\mathrm{UL}$ & {$[0]$} & & & & 7.96 & [3][4] \\
\hline NGC 4374 & $\mathrm{~L}$ & 38.97 & & {$[0]$} & & & & 9.07 & {$[1][2][3][4][5]$} \\
\hline NGC 4378 & $\mathrm{~S}$ & 37.52 & $\mathrm{UL}$ & {$[0]$} & & & & 8.06 & {$[3][4]$} \\
\hline NGC 4388 & $\mathrm{~S}$ & 37.05 & & {$[0]$} & 40.70 & UL & {$[8]$} & 6.80 & {$[3][4][5]$} \\
\hline NGC 4394 & $\mathrm{~L}$ & 36.66 & UL & {$[0]$} & & & & 7.19 & {$[3][4]$} \\
\hline NGC 4395 & $\mathrm{~S}$ & 35.32 & $\mathrm{UL}$ & {$[0]$} & 39.60 & UL & {$[8]$} & 4.83 & {$[1][3][4][5]$} \\
\hline NGC 4414 & $\mathrm{~T}$ & 36.19 & $\mathrm{UL}$ & {$[0]$} & & & & 7.02 & [3][4] \\
\hline NGC 4419 & $\mathrm{~T}$ & 37.14 & & {$[0]$} & & & & 6.96 & [3] $[4]$ \\
\hline NGC 4429 & $\mathrm{~T}$ & 36.75 & UL & {$[0]$} & & & & 7.90 & [3] $[4]$ \\
\hline NGC 4435 & $\mathrm{~T} / \mathrm{H}$ & 36.75 & $\mathrm{UL}$ & {$[0]$} & & & & 7.68 & {$[3][4]$} \\
\hline NGC 4438 & $\mathrm{~L}$ & 36.66 & UL & $0]$ & & & & 7.40 & {$[3][4]$} \\
\hline NGC 4450 & $\mathrm{~L}$ & 37.01 & & {$[0]$} & & & & 7.35 & {$[3][4][5]$} \\
\hline NGC 4457 & $\mathrm{~L}$ & 36.74 & UL & {$[0]$} & & & & 7.02 & {$[3][4][5]$} \\
\hline NGC 4459 & $\mathrm{~T}$ & 36.71 & $\mathrm{UL}$ & $0]$ & & & & 7.86 & {$[3][4]$} \\
\hline NGC 4472 & $\mathrm{~S}$ & 37.28 & & {$[0]$} & & & & 8.78 & {$[3][4][5]$} \\
\hline NGC 4477 & $\mathrm{~S}$ & 36.71 & UL & {$[0]$} & & & & 7.87 & {$[3][4]$} \\
\hline NGC 4486 (M87) & $\mathrm{L}$ & 40.14 & & {$[0]$} & 39.91 & & [10] & 9.33 & [1] [3][4][5] \\
\hline NGC 4486 (M87) & $\mathrm{L}$ & 40.14 & & {$[0]$} & 41.09 & & {$[10]$} & 9.33 & {$[1][3][4][5]$} \\
\hline NGC 4494 & $\mathrm{~L}$ & 36.13 & UL & {$[0]$} & & & & 7.60 & {$[3][4][5]$} \\
\hline NGC 4501 & $\mathrm{~S}$ & 36.75 & UL & {$[0]$} & & & & 7.83 & {$[3][4][5]$} \\
\hline NGC 4527 & $\mathrm{~T}$ & 36.56 & $\mathrm{UL}$ & {$[0]$} & & & & 7.38 & {$[3][4]$} \\
\hline
\end{tabular}




\begin{tabular}{|c|c|c|c|c|c|c|c|c|c|}
\hline $\begin{array}{l}\text { Sources } \\
(1)\end{array}$ & $\begin{array}{c}\text { Type } \\
(2)\end{array}$ & $\begin{array}{c}\log _{10}\left(L_{R}[\mathrm{erg} / \mathrm{s}]\right) \\
(3)\end{array}$ & $\begin{array}{c}\text { Notes } \\
(4)\end{array}$ & $\begin{array}{l}\text { Ref. } \\
(5)\end{array}$ & $\begin{array}{c}\log _{10}\left(L_{\gamma}[\mathrm{erg} / \mathrm{s}]\right) \\
(6)\end{array}$ & $\begin{array}{l}\text { Notes } \\
(7)\end{array}$ & $\begin{array}{l}\text { Ref. } \\
(8)\end{array}$ & $\begin{array}{c}\log _{10}\left(M / M_{\odot}\right) \\
(9)\end{array}$ & $\begin{array}{l}\text { Ref. } \\
(10)\end{array}$ \\
\hline NGC 4548 & $\mathrm{~L}$ & 36.79 & & {$[0]$} & & & & 7.32 & $\begin{array}{l}3][4][5] \\
\end{array}$ \\
\hline NGC 4550 & $\mathrm{~L}$ & 36.55 & & {$[0]$} & & & & 6.88 & {$[3][4]$} \\
\hline NGC 4552 & $\mathrm{~T}$ & 38.47 & & {$[0]$} & & & & 8.57 & [3][4] \\
\hline NGC 4565 & $\mathrm{~S}$ & 36.80 & & {$[0]$} & & & & 7.64 & {$[3][4][5]$} \\
\hline NGC 4569 & $\mathrm{~T}$ & 36.75 & UL & 0 & & & & 7.45 & [3][4] \\
\hline NGC 4579 & $\mathrm{~S} / \mathrm{L}$ & 38.15 & & {$[0]$} & & & & 7.81 & {$[3][4][5]$} \\
\hline NGC 4589 & $\mathrm{~L}$ & 38.28 & & {$[0]$} & & & & 8.35 & {$[3][4]$} \\
\hline NGC 4596 & $\mathrm{~L}$ & 36.75 & UL & {$[0]$} & & & & 7.54 & {$[3][4]$} \\
\hline NGC 4636 & $\mathrm{~L}$ & 36.92 & & 0 & & & & 8.09 & {$[3][4][5]$} \\
\hline NGC 4639 & $\mathrm{~S}$ & 36.75 & UL & {$[0]$} & & & & 6.60 & [3][4] \\
\hline NGC 4643 & $\mathrm{~T}$ & 37.08 & UL & {$[0]$} & & & & 7.58 & {$[3][4]$} \\
\hline NGC 4651 & $\mathrm{~L}$ & 36.75 & UL & {$[0]$} & & & & 6.84 & [3] [4] \\
\hline NGC 4698 & $\mathrm{~S}$ & 36.71 & $\mathrm{UL}$ & {$[0]$} & & & & 7.48 & [3] [4] \\
\hline NGC 4713 & $\mathrm{~T}$ & 36.81 & UL & {$[0]$} & & & & 4.14 & [3] [4] \\
\hline NGC 4725 & $\mathrm{~S}$ & 36.40 & UL & {$[0]$} & & & & 7.40 & {$[3][4][5]$} \\
\hline NGC 4736 & $\mathrm{~L}$ & 35.80 & & {$[0]$} & & & & 7.12 & [3][4][5] \\
\hline NGC 4750 & $\mathrm{~L}$ & 37.27 & UL & {$[0]$} & & & & 7.40 & {$[3][4]$} \\
\hline NGC 4762 & $\mathrm{~L}$ & 36.66 & & {$[0]$} & & & & 7.54 & [3] [4] \\
\hline NGC 4772 & $\mathrm{~L}$ & 37.20 & & {$[0]$} & & & & 7.55 & [3] 4$]$ \\
\hline NGC 4826 & $\mathrm{~T}$ & 35.44 & UL & {$[0]$} & & & & 6.84 & [3] 4$]$ \\
\hline NGC 4866 & $\mathrm{~L}$ & 36.71 & UL & 0 & & & & 8.20 & [3] 4$]$ \\
\hline NGC 5005 & $\mathrm{~L}$ & 36.96 & UL & {$[0]$} & & & & 7.84 & [3] [4] \\
\hline NGC 5012 & $\mathrm{~T}$ & 37.47 & UL & [0] & & & & 7.46 & [3] [4] \\
\hline NGC 5033 & $\mathrm{~S}$ & 36.95 & & {$[0]$} & & & & 7.36 & {$[3][4][5]$} \\
\hline NGC 5055 & $\mathrm{~T}$ & 36.01 & UL & {$[0]$} & & & & 6.86 & [3][4] \\
\hline NGC 5194 & $\mathrm{~S}$ & 36.07 & UL & {$[0]$} & & & & 6.74 & {$[3][4][5]$} \\
\hline NGC 5195 & $\mathrm{~L}$ & 36.24 & UL & {$[0]$} & & & & 7.28 & {$[3][4]$} \\
\hline NGC 5273 & $\mathrm{~S}$ & 36.96 & UL & {$[0]$} & & & & 6.20 & {$[3][4][5]$} \\
\hline NGC 5297 & $\mathrm{~L}$ & 37.59 & $\mathrm{UL}$ & {$[0]$} & & & & 6.60 & [3][4] \\
\hline NGC 5322 & $\mathrm{~L}$ & 38.36 & & [0] & & & & 8.43 & [3] [4] \\
\hline NGC 5353 & $\mathrm{~L} / \mathrm{T}$ & 38.68 & & {$[0]$} & & & & 8.82 & [3] [4] \\
\hline NGC 5354 & $\mathrm{~T} / \mathrm{L}$ & 38.28 & & {$[0]$} & & & & 8.27 & [3] [4] \\
\hline NGC 5363 & $\mathrm{~L}$ & 38.54 & & 0 & & & & 8.33 & [3] 4$]$ \\
\hline NGC 5371 & $\mathrm{~L}$ & 37.59 & UL & {$[0]$} & & & & 7.92 & [3] 4$]$ \\
\hline NGC 5377 & $\mathrm{~L}$ & 37.72 & & {$[0]$} & & & & 7.87 & [3] 4$]$ \\
\hline NGC 5395 & $\mathrm{~S} / \mathrm{L}$ & 37.77 & UL & {$[0]$} & & & & 7.53 & [3] 4$]$ \\
\hline NGC 5448 & $\mathrm{~L}$ & 37.46 & UL & 0 & & & & 7.24 & [3] 4$]$ \\
\hline NGC 5485 & $\mathrm{~L}$ & 37.47 & UL & {$[0]$} & & & & 8.08 & [3] [4] \\
\hline NGC 5566 & L & 37.14 & UL & [0] & & & & 7.70 & [3] 4$]$ \\
\hline NGC 5631 & $\mathrm{~S} / \mathrm{L}$ & 37.46 & UL & {$[0]$} & & & & 7.77 & [3] 4$]$ \\
\hline NGC 5656 & $\mathrm{~T}$ & 37.52 & $\mathrm{UL}$ & {$[0]$} & & & & 7.11 & [3] [4] \\
\hline NGC 5678 & $\mathrm{~T}$ & 37.36 & UL & {$[0]$} & & & & 7.35 & [3] [4] \\
\hline NGC 5701 & $\mathrm{~T}$ & 37.13 & UL & {$[0]$} & & & & 7.22 & [3] [4] \\
\hline NGC 5746 & $\mathrm{~T}$ & 37.19 & UL & [0] & & & & 8.02 & [3] [4] \\
\hline NGC 5813 & $\mathrm{~L}$ & 37.51 & & {$[0]$} & & & & 8.45 & [3] 4$]$ \\
\hline NGC 5838 & $\mathrm{~T}$ & 37.37 & & {$[0]$} & & & & 8.74 & [3] [4] \\
\hline NGC 5846 & $\mathrm{~T}$ & 37.97 & & {$[0]$} & & & & 8.44 & [3] [4] \\
\hline NGC 5866 & $\mathrm{~T}$ & 37.48 & & {$[0]$} & & & & 7.78 & [3] [4] \\
\hline NGC 5879 & $\mathrm{~T} / \mathrm{L}$ & 36.75 & UL & 0 & & & & 6.45 & [3] [4] \\
\hline NGC 5921 & $\mathrm{~T}$ & 37.06 & UL & {$[0]$} & & & & 6.50 & [3] [4] \\
\hline NGC 6340 & $\mathrm{~L}$ & 37.12 & UL & {$[0]$} & & & & 7.48 & [3] $[4]$ \\
\hline NGC 6384 & $\mathrm{~T}$ & 37.11 & UL & {$[0]$} & & & & 7.25 & [3] [4] \\
\hline NGC 6482 & $\mathrm{~T} / \mathrm{S}$ & 37.70 & UL & 0 & & & & 9.04 & [3] [4] \\
\hline NGC 6500 & $\mathrm{~L}$ & 39.38 & & [0] & & & & 8.23 & {$[3][4][5]$} \\
\hline NGC 6503 & $\mathrm{~T} / \mathrm{S}$ & 35.83 & UL & {$[0]$} & & & & 5.39 & {$[3][4]$} \\
\hline NGC 6703 & $\mathrm{~L}$ & 37.54 & UL & {$[0]$} & & & & 7.90 & [3] $[4]$ \\
\hline NGC 6951 & $\mathrm{~S}$ & 37.20 & $\mathrm{UL}$ & {$[0]$} & & & & 7.11 & {$[3][4]$} \\
\hline NGC 7177 & $\mathrm{~T}$ & 36.82 & UL & {$[0]$} & & & & 7.28 & [3] [4] \\
\hline NGC 7217 & $\mathrm{~L}$ & 36.41 & UL & {$[0]$} & & & & 7.41 & [3] [4] \\
\hline NGC 7331 & $\mathrm{~T}$ & 36.61 & UL & {$[0]$} & & & & 7.41 & [3] [4] \\
\hline NGC 7479 & $\mathrm{~S}$ & 37.66 & & {$[0]$} & & & & 7.60 & {$[3][4]$} \\
\hline NGC 7626 & L & 39.18 & & [0] & & & & 8.68 & [3] 4$]$ \\
\hline NGC 7742 & $\mathrm{~T} / \mathrm{S}$ & 36.99 & UL & {$[0]$} & & & & 6.45 & {$[3][4]$} \\
\hline NGC 7743 & $\mathrm{~S}$ & 37.03 & & {$[0]$} & & & & 6.50 & {$[3][4][5]$} \\
\hline NGC 7814 & $\mathrm{~L}$ & 36.66 & UL & [0] & & & & 7.83 & {$[3][4]$} \\
\hline Ark 564 & S & 38.59 & & [5] & & & & 6.50 & [5] \\
\hline Cyg A & $\mathrm{S} / \mathrm{L}$ & 41.43 & & {$[5]$} & & & & 9.40 & {$[5]$} \\
\hline Fairall 9 & $\mathrm{~S}$ & 37.68 & UL & [5] & 42.70 & UL & [8] & 7.91 & [5] \\
\hline IC 1459 & $\mathrm{~L}$ & 39.71 & & {$[5]$} & & & & 8.88 & {$[2][3][4][5]$} \\
\hline IC 4296 & $\mathrm{~L}$ & 39.68 & & [5] & & & & 9.10 & [3][4][5] \\
\hline IC 4329A & $\mathrm{S}$ & 38.94 & & {$[5]$} & 42.40 & UL & [8] & 8.26 & {$[3][4][5]$} \\
\hline Mrk 3 & $\mathrm{~S}$ & 39.86 & & {$[5]$} & & & & 8.81 & [5] \\
\hline Mrk 78 & $\mathrm{~S}$ & 39.86 & & {$[5]$} & & & & 7.92 & [5] \\
\hline Mrk 279 & $\mathrm{~S}$ & 38.78 & & {$[5]$} & 42.70 & UL & [8] & 7.62 & {$[5]$} \\
\hline Mrk 335 & $\mathrm{~S}$ & 38.27 & & {$[5]$} & & & & 6.79 & [5] \\
\hline
\end{tabular}




\begin{tabular}{|c|c|c|c|c|c|c|c|c|c|}
\hline $\begin{array}{l}\text { Sources } \\
(1)\end{array}$ & $\begin{array}{c}\text { Type } \\
(2)\end{array}$ & $\begin{array}{c}\log _{10}\left(L_{R}[\mathrm{erg} / \mathrm{s}]\right) \\
(3)\end{array}$ & $\begin{array}{c}\text { Notes } \\
(4)\end{array}$ & $\begin{array}{c}\text { Ref. } \\
(5)\end{array}$ & $\begin{array}{c}\log _{10}\left(L_{\gamma}[\mathrm{erg} / \mathrm{s}]\right) \\
(6)\end{array}$ & $\begin{array}{c}\text { Notes } \\
(7)\end{array}$ & $\begin{array}{c}\text { Ref. } \\
(8)\end{array}$ & $\begin{array}{c}\log _{10}\left(M / M_{\odot}\right) \\
(9)\end{array}$ & $\begin{array}{l}\text { Ref. } \\
(10)\end{array}$ \\
\hline Mrk 348 & $\mathrm{~S}$ & 39.70 & & {$[5]$} & 41.60 & UL & {$[8]$} & 7.17 & {$[5]$} \\
\hline Mrk 478 & $\mathrm{~S}$ & 38.75 & & {$[5]$} & & & & 7.30 & $5]$ \\
\hline Mrk 507 & $\mathrm{~S}$ & 38.78 & & {$[5]$} & & & & 7.10 & 5 \\
\hline Mrk 509 & $\mathrm{~S}$ & 38.30 & & {$[5]$} & 42.60 & UL & [8] & 7.86 & {$[5]$} \\
\hline Mrk 573 & S & 38.22 & & {$[5]$} & & & & 7.25 & $5]$ \\
\hline Mrk 590 & $\mathrm{~S}$ & 38.70 & & {$[5]$} & & & & 7.23 & $5]$ \\
\hline Mrk 766 & $\mathrm{~S}$ & 38.32 & & {$[5]$} & & & & 6.64 & 5 \\
\hline Mrk 1066 & $\mathrm{~S}$ & 38.68 & & {$[5]$} & & & & 6.93 & {$[5]$} \\
\hline NGC 1052 & $\mathrm{~L}$ & 39.86 & & {$[5]$} & & & & 8.22 & [3][4][5] \\
\hline NGC 1068 & $\mathrm{~S}$ & 39.12 & & {$[5]$} & & & & 7.78 & [1][2][3][4][5] \\
\hline NGC 1365 & $\mathrm{~S}$ & 38.80 & & {$[5]$} & 40.90 & $\mathrm{UL}$ & [8] & 7.62 & $\begin{array}{l}{[3][4][5]} \\
\text { [a] }\end{array}$ \\
\hline NGC 1386 & $\mathrm{~S}$ & 36.70 & & {$[5]$} & & & & 7.65 & [3][4][5] \\
\hline NGC 1667 & S & 37.34 & & {$[5]$} & & & & 7.97 & {$[3][4][5]$} \\
\hline NGC 2110 & $\mathrm{~S}$ & 38.99 & & {$[5]$} & 41.40 & $\mathrm{UL}$ & {$[8]$} & 8.53 & {$[3][4][5]$} \\
\hline NGC 2992 & $\mathrm{~S}$ & 38.64 & & {$[5]$} & & & & 7.76 & {$[3][4][5]$} \\
\hline NGC 3362 & $\mathrm{~S}$ & 38.47 & & {$[5]$} & & & & 7.01 & [3] $[4][5]$ \\
\hline NGC 4117 & $\mathrm{~S}$ & 35.70 & & {$[5]$} & & & & 6.65 & [3][4][5] \\
\hline NGC 4594 & $\mathrm{~L}$ & 37.84 & & $5]$ & & & & 8.83 & [1][3][4][5] \\
\hline NGC 5252 & $\mathrm{~S}$ & 38.96 & & {$[5]$} & 42.20 & $\mathrm{UL}$ & {$[8]$} & 8.10 & [3][4][5] \\
\hline NGC 5347 & $\mathrm{~S}$ & 37.10 & & [5] & & & & 6.70 & [3] $[4][5]$ \\
\hline NGC 5548 & $\mathrm{~S}$ & 38.58 & & {$[5]$} & 42.10 & UL & [8] & 8.08 & [3] 4$][5]$ \\
\hline NGC 5929 & $\mathrm{~S}$ & 38.30 & & {$[5]$} & & & & 7.19 & [3][4][5] \\
\hline NGC 6166 & $\mathrm{~S}$ & 39.95 & & 5 & & & & 9.04 & [3][4][5] \\
\hline NGC 6251 & $\mathrm{~S}$ & 40.93 & & {$[5]$} & & & & 8.93 & {$[2][3][4][5]$} \\
\hline NGC 7469 & $\mathrm{~S}$ & 38.38 & & {$[5]$} & 41.80 & $\mathrm{UL}$ & [8] & 7.24 & [3][4][5] \\
\hline NGC 7672 & S & $\begin{array}{l}30.00 \\
37.25\end{array}$ & & 5 & & & {$[0]$} & 6.80 & {$[3][4][5]$} \\
\hline NGC 7682 & $\mathrm{~S}$ & 38.88 & & $5]$ & & & & 7.25 & [3] $[4][5]$ \\
\hline $3 \mathrm{C} 120$ & $\mathrm{~S}$ & 41.55 & & {$[5]$} & & & & 7.36 & {$\left[\begin{array}{l}{[5]} \\
{[5]}\end{array}\right.$} \\
\hline 3C 390.3 & $\mathrm{~S}$ & 41.09 & & {$[5]$} & & & & 8.53 & {$[5]$} \\
\hline UGC 6100 & $\mathrm{~S}$ & 38.50 & & 5 & & & & 7.72 & {$[5]$} \\
\hline NGC 5128 (Cen A) & $\mathrm{S}$ & 38.67 & & {$[11]$} & 38.49 & & {$[12]$} & 7.70 & {$[11]$} \\
\hline NGC 5128 (Cen A) & $\mathrm{S}$ & 38.67 & & {$[11]$} & 40.61 & & $12]$ & 7.70 & {$[11]$} \\
\hline IC 310 & Galaxy & 39.77 & & {$[15]$} & 41.70 & & {$[14]$} & 8.30 & {$[14]$} \\
\hline IC 310 & Galaxy & 39.77 & & {$[15]$} & 43.30 & & {$[14]$} & $\begin{array}{l}0.50 \\
8.30\end{array}$ & {$[14]$} \\
\hline Cgy-X1 1 & GBH & 29.66 & & {$[5]$} & 32.45 & & {$[16]$} & 1.06 & {$[5]$} \\
\hline Cgy-X1 & GBH & 29.66 & & $5]$ & 33.56 & & {$[16]$} & 1.06 & {$[5]$} \\
\hline Cgy-X3 & GBH & 32.17 & & $5]$ & 33.67 & & {$[17]$} & 1.00 & $5]$ \\
\hline Cgy-X3 & GBH & 32.17 & & $5]$ & 36.37 & & {$[17]$} & 1.00 & {$[5]$} \\
\hline GRO J1655-40 & GBH & 29.94 & & 5 & & & & 0.85 & 5 \\
\hline GRS $1915+105$ & GBH & 31.76 & & 5 & & & & 1.20 & 5 \\
\hline GX 339-4 & GBH & 29.91 & & {$[5]$} & & & & 1.00 & {$[5]$} \\
\hline LS 5039 & GBH & 30.09 & & $5]$ & & & & 1.00 & {$[5]$} \\
\hline XTE J1118+480 & GBH & 28.92 & & 5 & & & & 1.00 & {$[5]$} \\
\hline XTE J1859+226 & GBH & 29.18 & & 5 & & & & 0.88 & 5 \\
\hline XTE J1550-564 & $\mathrm{GBH}$ & 31.79 & & {$[6]$} & & & & 0.98 & [7] \\
\hline
\end{tabular}

Column (1): Source name; Column (2): source spectral class: L - LINER; S - Seyfert; H - HII region spectral type; T: source with transition spectrum (LINER+HII); GBH: galactic black hole binary (or microquasar) (for more details see Merloni et al. 2003; (Nagar et al. 2005); Column (3): logarithm of the core radio luminosity (in erg/s); Column (6): logarithm of the gamma-ray luminosity (in erg/s); Column (9): logarithm of the black hole mass (in solar units); Columns (4) and (7): upper limit of the core radio and gamma luminosity (UL); Columns (5), (8) and (10): References.

Table 2 Radio and gamma-ray emission of blazars.

\begin{tabular}{|c|c|c|c|c|c|c|c|c|c|}
\hline $\begin{array}{c}\text { Sources } \\
(1)\end{array}$ & $\begin{array}{l}\text { Type } \\
(2)\end{array}$ & $\begin{array}{c}\log _{10}\left(L_{\gamma}^{\text {iso }}\right) \\
(3)\end{array}$ & $\begin{array}{c}\log _{10}\left(L_{\gamma}\right) \\
(4)\end{array}$ & $\begin{array}{l}\text { Ref. } \\
(5)\end{array}$ & $\begin{array}{c}\log _{10}\left(L_{R}^{i s o}\right) \\
(6)\end{array}$ & $\begin{array}{c}\log _{10}\left(L_{R}\right) \\
(7)\end{array}$ & $\begin{array}{c}\text { Ref. } \\
(8)\end{array}$ & $\begin{array}{c}\log _{10}\left(M / M_{\odot}\right) \\
(9)\end{array}$ & $\begin{array}{l}\text { Ref. } \\
(10)\end{array}$ \\
\hline PKS $0754+100$ & BLL & 45.72 & 42.75 & {$[18]$} & 42.80 & 39.83 & [19] & 8.21 & {$[20]$} \\
\hline PKS $0823+033$ & BLL & 45.86 & 43.73 & {$[18]$} & 43.26 & 41.12 & [19] & 8.55 & {$[20]$} \\
\hline PKS $0829+046$ & BLL & 45.68 & 43.60 & {$[18]$} & 41.97 & 39.89 & {$[19]$} & 8.52 & $20]$ \\
\hline OJ 287 & BLL & 46.12 & 43.89 & {$[18]$} & 42.82 & 40.59 & [19] & 8.50 & [20] \\
\hline PKS 2155-304 & BLL & 45.98 & 43.80 & [18] & & & & 7.60 & [20] \\
\hline $4 \mathrm{C}-02.81$ & BLL & 47.18 & 44.59 & {$[18]$} & 44.28 & 41.69 & [19] & 9.96 & {$[20]$} \\
\hline 3C 454.3 & FSRQ & 48.79 & 45.91 & {$[18]$} & 44.85 & 41.97 & {$[19]$} & 6.76 & $20]$ \\
\hline S3 $2141+17$ & FSRQ & 46.01 & 43.82 & {$[18]$} & & & & 8.14 & {$[20]$} \\
\hline AO $0235+164$ & BLL & 47.38 & 44.72 & [18] & 43.98 & 41.32 & [19] & 7.98 & [20] \\
\hline $4 C+28.07$ & FSRQ & 47.78 & 45.27 & {$[18]$} & 44.44 & 41.92 & [19] & 7.98 & {$[20]$} \\
\hline MKN 421 & BLL & 44.88 & 43.08 & {$[18]$} & & & & 8.23 & $20]$ \\
\hline PKS 1127-145 & FSRQ & 47.68 & 44.92 & {$[18]$} & 44.71 & 41.95 & [19] & 7.75 & [20] \\
\hline $4 C+29.45$ & FSRQ & 47.31 & 44.21 & [18] & 43.72 & 40.62 & [19] & 8.56 & {$[20]$} \\
\hline ON 231 & BLL & 45.18 & 43.28 & {$[18]$} & & & & 8.01 & [20] \\
\hline $4 \mathrm{C}+21.35$ & FSRQ & 47.50 & 43.90 & {$[18]$} & 43.02 & 39.41 & [19] & 8.18 & {$[20]$} \\
\hline 3 C 273 & FSRQ & 46.34 & 43.76 & {$[18]$} & 43.51 & 40.93 & [19] & 9.38 & [20] \\
\hline $3 \mathrm{C} 279$ & FSRQ & 47.64 & 44.70 & {$[18]$} & 44.22 & 41.28 & [19] & 7.82 & {$[20]$} \\
\hline
\end{tabular}




\begin{tabular}{|c|c|c|c|c|c|c|c|c|c|}
\hline $\begin{array}{c}\text { Sources } \\
(1)\end{array}$ & $\begin{array}{c}\text { Type } \\
(2)\end{array}$ & $\begin{array}{c}\log _{10}\left(L_{\gamma}^{i s o}\right) \\
(3)\end{array}$ & $\begin{array}{c}\log _{10}\left(L_{\gamma}\right) \\
(4) \\
\end{array}$ & $\begin{array}{l}\text { Ref. } \\
(5)\end{array}$ & $\begin{array}{c}\log _{10}\left(L_{R}^{i s o}\right) \\
(6)\end{array}$ & $\begin{array}{c}\log _{10}\left(L_{R}\right) \\
(7)\end{array}$ & $\begin{array}{c}\text { Ref. } \\
(8)\end{array}$ & $\begin{array}{c}\log _{10}(M / M \odot) \\
(9) \\
\end{array}$ & $\begin{array}{l}\text { Ref. } \\
(10)\end{array}$ \\
\hline PG $1424+240$ & BLL & 45.98 & 43.80 & [18] & & & & 6.42 & {$[20]$} \\
\hline AP Lib & BLL & 44.50 & 42.83 & {$[18]$} & & & & 8.64 & {$[20]$} \\
\hline PKS 1510-089 & FSRQ & 47.44 & 44.51 & {$[18]$} & 42.95 & 40.02 & {$[19]$} & 8.31 & {$[20]$} \\
\hline NRAO 530 & FSRQ & 47.39 & 43.45 & [18] & 44.54 & 40.61 & [19] & 8.07 & {$[20]$} \\
\hline OT 081 & BLL & 46.26 & 44.16 & [18] & 42.69 & 40.59 & [19] & 8.64 & [20] \\
\hline $4 \mathrm{C}+10.45$ & FSRQ & 47.56 & 44.70 & {$[18]$} & 44.22 & 41.37 & {$[19]$} & 8.07 & {$[20]$} \\
\hline $3 \mathrm{C} 66 \mathrm{~A}$ & BLL & 47.31 & 44.67 & {$[18]$} & & & & 8.30 & $20]$ \\
\hline PKS $1604+159$ & BLL & 46.06 & 43.86 & {$[18]$} & & & & 8.25 & [20] \\
\hline B2 $1811+31$ & BLL & 44.85 & 43.06 & [18] & & & & 8.82 & [20] \\
\hline OS 319 & FSRQ & 46.98 & 44.88 & [18] & 44.69 & 42.58 & [19] & 8.22 & [20] \\
\hline $4 \mathrm{C}+38.41$ & FSRQ & 48.72 & 45.44 & {$[18]$} & 44.85 & 41.56 & $19]$ & 7.54 & {$[20]$} \\
\hline MKN 501 & BLL & 44.46 & 42.80 & [18] & & & & 8.72 & [20] \\
\hline PKS 0454-46 & FSRQ & 47.11 & 44.54 & [18] & & & & 8.05 & [20] \\
\hline $4 \mathrm{C}+56.27$ & BLL & 46.93 & 44.61 & {$[18]$} & 43.40 & 41.08 & [19] & 9.10 & {$[20]$} \\
\hline S5 $1803+784$ & BLL & 47.08 & 44.83 & {$[18]$} & 43.75 & 41.50 & {$[19]$} & 8.82 & {$[20]$} \\
\hline BL Lac & BLL & 45.16 & 43.40 & {$[18]$} & 41.50 & 39.73 & {$[19]$} & 8.58 & {$[20]$} \\
\hline $4 \mathrm{C}+51.37$ & FSRQ & 47.83 & 45.01 & [18] & 44.42 & 41.61 & [19] & 7.97 & [20] \\
\hline PKS 2052-47 & FSRQ & 48.33 & 45.34 & {$[18]$} & & & & 7.88 & $20]$ \\
\hline S5 $0716+714$ & BLL & 46.78 & 44.46 & {$[18]$} & 42.44 & 40.12 & {$[19]$} & 7.74 & {$[20]$} \\
\hline EXO $0706.1+5913$ & BLL & 44.70 & 42.96 & {$[18]$} & & & & 8.67 & {$[20]$} \\
\hline $4 \mathrm{C}+71.07$ & FSRQ & 48.36 & 45.16 & [18] & 45.23 & 42.03 & [19] & 7.22 & [20] \\
\hline B2 $0827+24$ & FSRQ & 47.17 & 44.07 & {$[18]$} & 43.68 & 40.58 & {$[19]$} & 8.41 & {$[20]$} \\
\hline MKN 180 & BLL & 43.85 & 42.40 & {$[18]$} & & & & 8.10 & $20]$ \\
\hline $1 \mathrm{ES} 1028+511$ & BLL & 45.76 & 43.66 & {$[18]$} & & & & 8.70 & 20 \\
\hline S4 $0954+658$ & BLL & 45.98 & 43.80 & {$[18]$} & & & & 8.37 & {$[20]$} \\
\hline 1ES $0806+524$ & BLL & 45.14 & 43.25 & {$[18]$} & & & & 8.65 & [20] \\
\hline $4 \mathrm{C}+55.17$ & FSRQ & 47.70 & 44.93 & {$[18]$} & & & & 8.42 & {$[20]$} \\
\hline PG $1246+586$ & BLL & 47.20 & 44.60 & {$[18]$} & & & & 9.15 & 20 \\
\hline S4 $0814+425$ & BLL & 46.17 & 45.01 & [18] & 42.41 & 41.24 & [19] & 8.01 & [20] \\
\hline S4 $0917+44$ & FSRQ & 48.72 & 45.60 & {$[18]$} & & & & 7.88 & [20] \\
\hline PG $1437+398$ & BLL & 45.31 & 43.36 & {$[18]$} & & & & 8.95 & {$[20]$} \\
\hline PKS 0336-019 & FSRQ & 46.96 & 43.93 & {$[18]$} & 44.16 & 41.13 & {$[19]$} & 7.21 & 20 \\
\hline PKS 0420-01 & FSRQ & 47.62 & 45.22 & {$[18]$} & 44.24 & 41.84 & {$[19]$} & 8.04 & {$[20]$} \\
\hline PKS 0440-00 & FSRQ & 47.57 & 44.84 & {$[18]$} & & & & 7.23 & {$[20]$} \\
\hline $4 \mathrm{C}-02.19$ & FSRQ & 48.02 & 45.28 & [18] & 44.98 & 42.24 & [19] & 8.50 & {$[20]$} \\
\hline PKS $0528+134$ & FSRQ & 48.32 & 45.36 & {$[18]$} & 45.00 & 42.04 & {$[19]$} & 7.66 & {$[20]$} \\
\hline PKS 0537-441 & BLL & 48.25 & 45.29 & [18] & & & & 8.02 & [20] \\
\hline PKS $0735+17$ & BLL & 46.57 & 44.19 & {$[18]$} & 43.23 & 40.85 & [19] & 8.08 & {$[20]$} \\
\hline PKS 2201+04 & BLL & 43.11 & 41.91 & {$[18]$} & & & & 7.76 & {$[20]$} \\
\hline $1 \mathrm{ES} 1741+196$ & BLL & 44.18 & 42.62 & {$[18]$} & & & & 8.93 & {$[20]$} \\
\hline
\end{tabular}

Column (1): source name; Column (2): BLL: BL Lac objects; FSRQ: Flat Spectrum Radio Quasar; Column (3): logarithm of the isotropic gamma-ray luminosity (in erg/s); Column (4): logarithm of the gamma-ray luminosity (in erg/s) corrected by Doppler boosting; Column (6): logarithm of the isotropic radio luminosity (in erg/s); Column (7): logarithm of the radio luminosity (in erg/s) corrected by Doppler boosting; Column (9): logarithm of the black hole mass (in solar units); Columns (5), (8) and (10): References.

Table 3 Gamma-ray emission of GRBs.

\begin{tabular}{|c|c|c|c|c|c|c|c|}
\hline $\begin{array}{l}\text { Sources } \\
\text { (1) }\end{array}$ & $\begin{array}{c}\log _{10}\left(L_{\gamma}^{\text {iso }}[\mathrm{erg} / \mathrm{s}]\right) \\
(2)\end{array}$ & $\begin{array}{c}\log _{10}\left(L_{\gamma}[\mathrm{erg} / \mathrm{s}]\right) \\
(3)\end{array}$ & $\begin{array}{l}\text { Ref. } \\
(4)\end{array}$ & $\begin{array}{c}\text { Sources } \\
(5)\end{array}$ & $\begin{array}{c}\log _{10}\left(L_{\gamma}^{\text {iso }}[\mathrm{erg} / \mathrm{s}]\right) \\
(6)\end{array}$ & $\begin{array}{c}\log _{10}\left(L_{\gamma}[\mathrm{erg} / \mathrm{s}]\right) \\
(7)\end{array}$ & $\begin{array}{r}\text { Ref } \\
(8)\end{array}$ \\
\hline 90323 & 53.06 & 50.14 & {$[18]$} & 21004 & 51.55 & 49.93 & {$[18]$} \\
\hline 90328 & 51.47 & 48.9 & [18] & 31203 & 49.43 & 47.52 & {$[18]$} \\
\hline 090902B & 53.63 & 51 & {$[18]$} & 30329 & 50.82 & 48.42 & {$[18]$} \\
\hline 090926A & 53.46 & 51.55 & {$[18]$} & 50709 & 51.22 & 49.87 & {$[18]$} \\
\hline 81222 & 53.28 & 50.35 & {$[18]$} & 050820A & 53.13 & 50.95 & {$[18]$} \\
\hline 90424 & 51.52 & 49.69 & {$[18]$} & 50904 & 52.63 & 50.62 & {$[18]$} \\
\hline 90618 & 52.19 & 50.02 & {$[18]$} & 60218 & 46.09 & 46.01 & {$[18]$} \\
\hline 91020 & 51.65 & 49.51 & {$[18]$} & 60418 & 51.28 & 50.16 & {$[18]$} \\
\hline 91127 & 50.47 & 48.13 & {$[18]$} & 70125 & 52.61 & 51.03 & {$[18]$} \\
\hline 091208B & 50.8 & 48.7 & {$[18]$} & 080319B & 51.26 & 49.13 & {$[18]$} \\
\hline 970228 & 50.63 & 48.02 & {$[18]$} & 50505 & 51.86 & 48.66 & {$[18]$} \\
\hline 970508 & 50.86 & 49.71 & {$[18]$} & 50814 & 52.36 & 49.32 & {$[18]$} \\
\hline 970828 & 51.43 & 49.32 & {$[18]$} & 051109A & 50.79 & 48.02 & {$[18]$} \\
\hline 971214 & 52.49 & 50.16 & {$[18]$} & $051221 \mathrm{~A}$ & 50.7 & 49.02 & {$[18]$} \\
\hline 980613 & 50.43 & 48.81 & {$[18]$} & 60124 & 53.6 & 50.55 & {$[18]$} \\
\hline 980425 & 46.84 & 45.1 & {$[18]$} & 60614 & 49.73 & 48.05 & {$[18]$} \\
\hline 980703 & 51.19 & 49.47 & {$[18]$} & 60707 & 51.18 & 49.16 & {$[18]$} \\
\hline 990123 & 52.79 & 50.36 & {$[18]$} & 60814 & 52.96 & 50.24 & {$[18]$} \\
\hline 990510 & 51.91 & 49.14 & {$[18]$} & 61021 & 49.75 & 47.8 & {$[18]$} \\
\hline 990705 & 52.17 & 49.8 & {$[18]$} & $061222 \mathrm{~A}$ & 52.76 & 49.8 & {$[18]$} \\
\hline 991216 & 52.86 & 50.36 & {$[18]$} & 70306 & 52.7 & 50.16 & {$[18]$} \\
\hline 21004 & 52.54 & 50.39 & {$[18]$} & 70318 & 52.69 & 50.76 & {$[18]$} \\
\hline 926 & 53.82 & 51.58 & [18] & 70508 & 51.76 & 49.03 & {$[18]$} \\
\hline
\end{tabular}


22

\begin{tabular}{|c|c|c|c|c|c|c|c|}
\hline $\begin{array}{l}\text { Sources } \\
(1)\end{array}$ & $\begin{array}{c}\log _{10}\left(L_{\gamma}^{\text {iso }}[\operatorname{erg} / s]\right) \\
(2)\end{array}$ & $\begin{array}{c}\log _{10}\left(L_{\gamma}[\mathrm{erg} / \mathrm{s}]\right) \\
(3)\end{array}$ & $\begin{array}{l}\text { Ref. } \\
(4)\end{array}$ & $\begin{array}{l}\text { Sources } \\
(5)\end{array}$ & $\begin{array}{c}\log _{10}\left(L_{\gamma}^{\text {iso }}[\mathrm{erg} / \mathrm{s}]\right) \\
(6)\end{array}$ & $\begin{array}{c}\log _{10}\left(L_{\gamma}[\operatorname{erg} / s]\right) \\
(7)\end{array}$ & $\begin{array}{l}\text { Ref. } \\
(8)\end{array}$ \\
\hline 10222 & 52.46 & 49.65 & {$[18]$} & 80310 & 51.81 & 49.1 & {$[18]$} \\
\hline 11211 & 51.62 & 49.41 & {$[18]$} & 080413B & 52.61 & 50.35 & {$[18]$} \\
\hline 20405 & 51.49 & 49.45 & {$[18]$} & 90313 & 51.23 & 48.38 & {$[18]$} \\
\hline 20813 & 52.29 & 49.47 & {$[18]$} & 91018 & 50.04 & 47.57 & {$[18]$} \\
\hline
\end{tabular}

Columns (1) and (5): source name; Columns (2) and (6) logarithm of the isotropic gamma-ray luminosity (in erg/s); Column (3) and (7): logarithm of the gamma-ray luminosity (in erg/s) corrected by Doppler boosting; Columns (4) and (8): References.

Notes. References (for all Tables): [0] Nagar et al. (2005), [1] Richstone et al. (1998), [2] Gebhardt et al. (2000). [3] Merritt \& Ferrarese

(2001), [4] Tremaine et al. (2002), [5] Merloni et al. (2003), [6] Hannikainen et al. (2001), [7] Remillard \& McClintock (2006), [8]

Ackermann et al. (2012), [9] Middleton et al. (2008), [10] Abdo et al. (2009b), [11] Israel (1998), [12] Abdo et al. (2010), [13] Aleksić et al. (2014b), [14]|Aleksić et al. (2014a), [15] Kadler et al. (2012), [16] Malvshev et al. (2013), [17] Piano et al. (2012), [18] Nemmen et al. (2012),

[19] Kharb et al. (2010) and [20] Vovk \& Neronov (2013). 